\title{
Quantum Quenches in Extended Systems
}

\author{
Pasquale Calabrese ${ }^{1}$ and John Cardy ${ }^{2}$ \\ 1 Dipartimento di Fisica dell'Università di Pisa and INFN, Pisa, Italy \\ Institute for Theoretical Physics, University of Amsterdam, 1018 XE Amsterdam, The Netherlands. and \\ 2 Oxford University, Rudolf Peierls Centre for Theoretical Physics, \\ Oxford, United Kingdom \\ All Souls College, Oxford, United Kingdom
}

(Dated: October 23, 2018)

\begin{abstract}
We study in general the time-evolution of correlation functions in a extended quantum system after the quench of a parameter in the hamiltonian. We show that correlation functions in $d$ dimensions can be extracted using methods of boundary critical phenomena in $d+1$ dimensions. For $d=1$ this allows to use the powerful tools of conformal field theory in the case of critical evolution. Several results are obtained in generic dimension in the gaussian (mean-field) approximation. These predictions are checked against the real-time evolution of some solvable models that allows also to understand which features are valid beyond the critical evolution.

All our findings may be explained in terms of a picture generally valid, whereby quasiparticles, entangled over regions of the order of the correlation length in the initial state, then propagate with a finite speed through the system. Furthermore we show that the long-time results can be interpreted in terms of a generalized Gibbs ensemble. We discuss some open questions and possible future developments.
\end{abstract}

\section{INTRODUCTION}

Suppose that an extended quantum system in $d$ dimensions (for example a quantum spin system), is prepared at time $t=0$ in a pure state $\left|\psi_{0}\right\rangle$ which is the ground state of some hamiltonian $H_{0}$ (or, more generally, in a thermal state at a temperature less than the mass gap to the first excited state). For times $t>0$ the system evolves unitarily according to the dynamics given by a different hamiltonian $H$, which may be related to $H_{0}$ by varying a parameter such as an external field. This variation, or quench, is supposed to be carried out over a time scale much less than the inverse mass gap. How does the state $|\psi(t)\rangle=e^{-i H t}\left|\psi_{0}\right\rangle$ evolve? For a finite number of degrees of freedom, the system generically shows a periodic (or quasiperiodic) behavior with a period that typically increases when the number of degrees of freedom grows. This is the well-known phenomenon of quantum recurrence. However, in the thermodynamic limit this is no longer necessarily the case, and the natural question arises as to whether the system (or, rather, a macroscopically large subsystem) reaches a stationary state for very large times. To attack this question we consider the simpler question of how the correlation functions, expectation values of products of local observables, evolve and whether they reach constant values for large times.

This problem has its own theoretical interest, being a first step towards the understanding of equilibration in quantum systems. On the same fundamental level we can also ask whether the approach can provide a new tool for the characterization of the collective excitations in strongly correlated systems. However, until recently it has been considered a largely academic question, because the time scales over which most condensed matter systems can evolve coherently without coupling to the local environment are far too short, and the effects of dissipation and noise are inescapable. Recent developments of experimental tools for studying the behavior of ultra-cold atoms have revised completely this negative attitude. In fact, thanks to the phenomenon of Feshbach resonance it is possible to tune the coupling of an interacting system to essentially any value and in extremely short times. In addition, the coupling to dissipative degrees of freedom can be much weaker than in ordinary condensed matter systems. This has allowed the observation, among other things, of the collapse and revival of a Bose-Einstein condensate [1] and of the quenching of a spinor condensate [2]. Furthermore, using highly anisotropic optical lattices, it has been possible to build and study essentially one-dimensional systems [3], and, of direct interest for this paper, the coherent non-equilibrium dynamics of these integrable models has been measured [4]. (Other interesting experiments are considered in Refs. [5].) These striking experimental results have largely motivated the development of new numerical methods to study non-equilibrium dynamics, the most successful one being the time-dependent density matrix renormalization group (DMRG) [6].

On the purely theoretical side these kind of questions were first considered (as far as we are aware) in the seventies in the context of the quantum Ising-XY model in Refs. [7, 8] (see also [9-14] for recent developments). However, in the very last few years, after the previously mentioned experimental progress, the study of quantum quenches has been pursued in a systematic manner and a large number of results is nowadays available. To quote only a few examples, the models considered include several realizations of one-dimensional (1D) Bose gases [15-25], Luttinger liquids [26, 27], coupled 1D condensates [28], strongly correlated 1D fermions [29], and mean field fermion condensates [30, 31]. A 
closely related topic, that will not be considered here, concerns the formation of defects when crossing a critical point with changing the external parameter at a fixed rate, i.e. the so-called quantum Kibble-Zurek mechanism [32].

Most of these papers concern the exact or approximate solutions of very specific models. These are often strongly relevant, since in many cases they can be directly compared with existing experimental results or give very accurate predictions for future investigations. However, from the study of specific models it is difficult to draw general conclusions on the physics of quantum quenches. There have been at least two notable exceptions. In Ref. [15] it was conjectured that the asymptotic state at very large times can be described by a generalized Gibbs ensemble (for more details we refer the reader to section VI where this conjecture will be explicitly discussed in relation to some of our findings). In Ref. [33] we studied the time evolution after a quench in general, exploiting the path integral approach and the well-known mapping of the quantum problem to a classical one in $d+1$ dimensions. The translational invariance in the (imaginary) time direction is explicitly broken and thus the initial state plays the role of a boundary condition. This limits the range of applicability of field-theoretical methods, but when the hamiltonian $H$ is at or close to a quantum critical point we could use the renormalization group (RG) theory of boundary critical behavior (see, e.g., [34]). From this point of view, particularly powerful analytic results are available for $d=1$ because then the $1+1$-dimensional problem is described asymptotically by a boundary conformal field theory (BCFT) [35, 36].

The aim of this paper is twofold: on the one hand we give a detailed description of the methods and the results reported briefly in Ref. [33], and on the other we generalize these results and give some new physical insights. Several results, in fact, appear here for the first time. The paper is organized as follows. In Sec. II we introduce the pathintegral formalism that is applied in the following section III to one-dimensional critical systems by means of CFT. In Sec. IV we consider two simple 1D models whose non-equilibrium dynamics is exactly solvable to check the correctness of the previous results and to extend our findings to gapped and lattice systems. In Sec. V we generalize the method to higher dimensions. In the last section VI we analyze all our results and explain most of the general findings in quantum quenches by means of simple physical arguments. We broadly discuss some open questions and possible future developments.

\section{PATH INTEGRAL FORMULATION AND SURFACE CRITICALITY}

Let us consider a lattice quantum theory in $d$ space dimensions. The lattice spacing is $a$, and the lattice variables are labelled by a discrete vector variable $\mathbf{r}$. Time is considered to be continuous. The dynamics of the theory is described by the hamiltonian $H$. Suppose we prepare this system in a state $\left|\psi_{0}\right\rangle$ that is not an eigenstate of $H$ and unitarily evolve it according to $H$. The expectation value of a local operator $\mathcal{O}\left(\left\{\mathbf{r}_{i}\right\}\right)$ at time $t$ is

$$
\left\langle\mathcal{O}\left(t,\left\{\mathbf{r}_{i}\right\}\right)\right\rangle=\left\langle\psi_{0}\left|e^{i H t} \mathcal{O}\left(\left\{\mathbf{r}_{i}\right\}\right) e^{-i H t}\right| \psi_{0}\right\rangle .
$$

We modify this time-dependent expectation value as

$$
\left\langle\mathcal{O}\left(t,\left\{\mathbf{r}_{i}\right\}\right)\right\rangle=Z^{-1}\left\langle\psi_{0}\left|e^{i H t-\epsilon H} \mathcal{O}\left(\left\{\mathbf{r}_{i}\right\}\right) e^{-i H t-\epsilon H}\right| \psi_{0}\right\rangle,
$$

where we have included damping factors $e^{-\epsilon H}$ in such a way as to make the path integral representation of the expectation value absolutely convergent. The normalization factor $Z=\left\langle\psi_{0}\left|e^{-2 \epsilon H}\right| \psi_{0}\right\rangle$ ensures that the expectation value of the identity is one. At the end of the calculation we shall set $\epsilon$ to zero.

Eq. (2) may be represented by an analytically continued path integral in imaginary time over the field variables $\phi(\tau, \mathbf{r})$, with initial and final values weighted by the matrix elements with $\left|\psi_{0}\right\rangle$ :

$$
\int[d \phi(\tau, \mathbf{r})]\left\langle\psi_{0} \mid \phi\left(\tau_{2}, \mathbf{r}\right)\right\rangle\left\langle\phi\left(\tau_{1}, \mathbf{r}\right) \mid \psi_{0}\right\rangle e^{-\int_{\tau_{1}}^{\tau_{2}} L[\phi] d \tau}
$$

where $\int_{\tau_{1}}^{\tau_{2}} L[\phi] d \tau$ is the (euclidean) action. The operator $\mathcal{O}$ is inserted at $\tau=0$, and the width of the slab is $2 \epsilon . \tau_{1}$ and $\tau_{2}$ should be considered as real numbers during the calculation, and only at the end should they be continued to their effective values $\pm \epsilon-i t$. In this way we have reduced the real-time non-equilibrium evolution of a $d$ dimensional systems to the thermodynamics of a $d+1$ field theory in a slab geometry with the initial state $\left|\psi_{0}\right\rangle$ playing the role of boundary condition at both the borders of the slab. The validity of our results relies on the technical assumption that the leading asymptotic behavior given by field theory, which applies to the Euclidean region (large imaginary times), may simply be analytically continued to find the behavior at large real time.

Eq. (2) can be in principle used to describe the time evolution in any theory and for all possible initial conditions. Unfortunately, in confined geometries only a few field theories with very specific boundary conditions can be solved analytically in such a way to have results that can be continued from real to complex values. Some examples of these will be given in Sec. V. (An interesting case concerns integrable massive boundary field theories as for example the 
recent application to the dynamics of coupled 1D condensates in Ref. [28].) However, the treatment greatly simplifies if a system is at or close to a (quantum) phase transition. In fact, in this case, we can use the powerful tools of Renormalization Group (RG) theory of boundary critical phenomena (see, e.g. [34]). For example, in the case of a scalar order parameter (corresponding to the Ising universality class), the slab geometry of above is usually described by the action [34]

$$
S(\phi)=\int d^{d} r \int_{\tau_{1}}^{\tau_{2}} d \tau\left(\frac{1}{2}(\partial \phi)^{2}+\frac{m^{2}}{2} \phi^{2}+\frac{g}{4 !} \phi^{4}\right)+\int d^{d} r c\left[\phi\left(\tau=\tau_{1}, r\right)+\phi\left(\tau=\tau_{2}, r\right)\right],
$$

where $m^{2}$ measures the distance from criticality, $g>0$ ensures the stability in the broken-symmetry phase, and $c$ denotes the surface enhancement, i.e. the difference of the interactions on the surface with respect to the bulk. The value of $c$ depends on the boundary conditions. For example, the choice $c=+\infty$ forces the field to vanish on the boundary, thus corresponding to Dirichlet boundary conditions. On the other hand $c=-\infty$ forces the field to diverge at the boundary. Finite values of $c$ correspond to intermediate boundary conditions. According to the RG theory, the different boundary universality classes are characterized by the fixed point of $c$. A complete RG analysis (see, e.g.,[34]) shows that the possible fixed points of $c$ are $c^{*}=\infty, 0,-\infty$, with $c^{*}= \pm \infty$ stable and $c^{*}=0$ unstable. Any other value of $c$ flows under RG transformations to $\pm \infty$. These universality classes are called ordinary $\left(c^{*}=\infty\right)$, special $\left(c^{*}=0\right)$, and extraordinary $\left(c^{*}=-\infty\right)[34]$.

Thus, for the purpose of extracting the asymptotic behavior, as long as $\left|\psi_{0}\right\rangle$ is translationally invariant, we may replace it by the appropriate RG-invariant boundary state $\left|\psi_{0}^{*}\right\rangle$ to which it flows. The difference may be taken into account, to leading order, by assuming that the RG-invariant boundary conditions are not imposed at $\tau=\tau_{1}$ and $\tau_{2}$ but at $\tau=\tau_{1}-\tau_{0}$ and $\tau=\tau_{2}+\tau_{0}$. In the language of boundary critical behavior, $\tau_{0}$ is called the extrapolation length [34]. It characterizes the RG distance of the actual boundary state from the RG-invariant one. It is always necessary because scale-invariant boundary states are not in fact normalizable[36]. It is expected to be of the order of the typical time-scale of the dynamics near the ground state of $H_{0}$, that is the inverse gap $m_{0}^{-1}$. (This can be checked explicitly for a free field theory, see later). The effect of introducing $\tau_{0}$ is simply to replace $\epsilon$ by $\epsilon+\tau_{0}$. The limit $\epsilon \rightarrow 0+$ can now safely be taken, so the width of the slab is then taken to be $2 \tau_{0}$. For simplicity in the calculations, in the following we will consider the equivalent slab geometry between $\tau=0$ and $\tau=2 \tau_{0}$ with the operator $\mathcal{O}$ inserted at $\tau=\tau_{0}+i t$. This is illustrated in the left part of Fig. 1. In fact, in this geometry we can consider products of operators at different times $t_{j}$, by analytically continuing their labels to $\tau_{0}+i t_{j}$.

Let us discuss to what initial conditions the fixed points correspond. In the case of free boundary conditions, when the order parameter is unconstrained, the continuum limit forces it to vanish there, so it corresponds to the ordinary transition $(c=\infty)$. For a lattice model, this corresponds to a completely disordered initial state (e.g. for the Ising chain in a transverse field corresponds to infinite field). In contrast for non-vanishing fixed boundary conditions (e.g. + or - for Ising-like systems), the continuum limit makes the order parameter to diverge at the boundary, thus corresponding to the extraordinary transition $(c=-\infty)$.

\section{ONE SPACE DIMENSION AND CONFORMAL FIELD THEORY}

In this section we specialize the methods just introduced to the case when $H$ is at a quantum critical point whose long-distance behavior is given by a 1+1-dimensional CFT, with dispersion relation $\Omega_{k}=v|k|$. We set $v=1$ in the following. RG-invariant boundary conditions then correspond to conformally invariant boundary states. In this case the correlation functions are accessible through the powerful tools of boundary CFT.
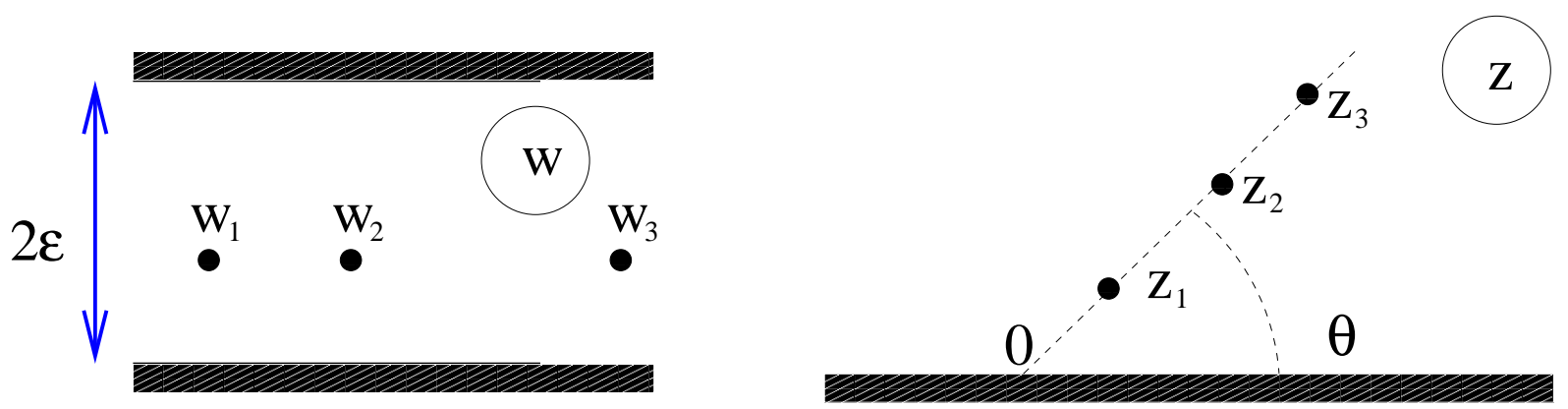

FIG. 1: Left: Space-imaginary time region in (2). $\operatorname{Im} w_{i}=\tau$, that will be analytically continued to $\tau \rightarrow \tau_{0}+i t$. Right: Conformal mapping of the left geometry to the upper half-plane (c.f. Eq. (5)). Note that $\arg z_{i}=\theta=\pi \tau / 2 \tau_{0}$. 
The main property we will repeatedly use in the following is the relation of correlation functions of (primary) operators among two geometries connected by a conformal transformation. For example, the slab geometry of above is just a two-dimensional strip whose points are labelled by a complex number $w=r+i \tau$ with $0<\operatorname{Im} w<2 \tau_{0}$. The strip can be obtained from the upper half-plane (UHP) $\operatorname{Im} z>0$ by the conformal mapping

$$
w(z)=\frac{2 \tau_{0}}{\pi} \log z
$$

with the images of points at the same imaginary time on the strip lying along $\arg z_{i}=\theta=\pi \tau / 2 \tau_{0}$. In the case where $\mathcal{O}$ is a product of local primary scalar operators $\Phi_{i}\left(w_{i}\right)$, the expectation value in the strip is related to the one in the UHP by the standard transformation

$$
\left\langle\prod_{i} \Phi_{i}\left(w_{i}\right)\right\rangle_{\mathrm{strip}}=\prod_{i}\left|w^{\prime}\left(z_{i}\right)\right|^{-x_{i}}\left\langle\prod_{i} \Phi_{i}\left(z_{i}\right)\right\rangle_{\mathrm{UHP}}
$$

where $x_{i}$ is the bulk scaling dimension of $\Phi_{i}$. Note that the (eventual) expectation values of the $\Phi_{i}$ in the ground state of $H$ are supposed to have been subtracted off. The asymptotic real time dependence is obtained via the analytic continuation $\tau \rightarrow \tau_{0}+i t$, and taking the limit $t, r_{i j} \gg \tau_{0}$.

In the following subsections we apply these methods to some specific cases.

\section{A. The one-point function}

In the UHP, the one-point function of a scalar primary field with bulk scaling dimension $x$ is $\langle\Phi(z)\rangle_{\mathrm{UHP}}=$ $A_{b}^{\Phi}[2 \operatorname{Im}(z)]^{-x}$, as a simple consequence of scaling invariance. The normalization factor $A_{b}^{\Phi}$ is a non-universal amplitude. In CFT the normalizations are chosen in such a way that the bulk two-point functions have unit amplitude (i.e. $\left\langle\Phi\left(z_{1}\right) \Phi\left(z_{2}\right)\right\rangle_{\text {bulk }}=\left|z_{2}-z_{1}\right|^{-2 x}$ ). This choice fixes unambiguously the amplitude $A_{b}^{\Phi}$ that turns out to depend both on the considered field $\Phi$ and on the boundary condition on the real axis $b$. It vanishes if $\Phi$ corresponds to an operator whose expectation value in $\left|\psi_{0}\right\rangle$ vanishes, and thus $\langle\Phi(t)\rangle=0$, for all times.

When the primary field is not vanishing on the boundary, performing the conformal mapping (5) we obtain

$$
\langle\Phi(w)\rangle_{\text {strip }}=\left|w^{\prime}(z)\right|^{-x}\langle\Phi(z(w))\rangle_{\mathrm{UHP}}=A_{b}^{\Phi}\left[\frac{\pi}{4 \tau_{0}} \frac{1}{\sin \left(\pi \tau /\left(2 \tau_{0}\right)\right)}\right]^{x}
$$

that continued to real time $\tau=\tau_{0}+i t$ gives

$$
\langle\Phi(t)\rangle=A_{b}^{\Phi}\left[\frac{\pi}{4 \tau_{0}} \frac{1}{\cosh \left(\pi t /\left(2 \tau_{0}\right)\right)}\right]^{x} \simeq A_{b}^{\Phi}\left(\frac{\pi}{2 \tau_{0}}\right)^{x} e^{-x \pi t / 2 \tau_{0}} .
$$

Thus the order parameter (and any other observables described by a primary field) decays exponentially in time to the ground-state value, with a non-universal relaxation time $t_{\text {rel }}^{\mathcal{O}}=2 \tau_{0} / x_{\mathcal{O}} \pi$. The ratio of the relaxation times of two different observables equals the inverse of the ratio of their scaling dimensions and it is then universal.

The normalization factor $A_{b}^{\Phi}$ is known for the simplest boundary universality classes [37]. In the case of $\Phi$ being the order parameter and the boundary condition is fixed $\left(\psi_{0}(x)=\infty\right) A_{b}^{\Phi}$ is 1 for the free boson and $2^{1 / 4}$ for the Ising model.

An important exception to this law is the local energy density (or any piece thereof). This corresponds to the $t t$ component of the energy-momentum tensor $T_{\mu \nu}$. In CFT this is not a primary operator. Indeed, if it is normalized so that $\left\langle T_{\mu \nu}\right\rangle_{\mathrm{UHP}}=0$, in the strip [38] $\left\langle T_{t t}(\mathbf{r}, \tau)\right\rangle=\pi c / 24\left(2 \tau_{0}\right)^{2}$ (where $c$ is the central charge of the CFT) so that it does not decay in time. Of course this is to be expected since the dynamics conserves energy. A similar feature is expected to hold for other local densities corresponding to globally conserved quantities which commute with $H$, for example the total spin in isotropic models.

\section{B. The two-point function}

In the case of the one-point function, scaling invariance was enough to fix the functional dependence on the position in the UHP. However, the form of the two-point function depends explicitly on the boundary universality class and on the operator considered. In the following subsections we will consider the equal-time correlation function for the order parameter in the gaussian and in the Ising universality classes that are easily treated in full generality. At the 
quantum level they describe (among the other things) a chain of harmonic oscillators (explicitly considered in Sec. IV A) and the Ising model in a transverse field (whose real time evolution has been considered in Refs. [7-9, 11] and is briefly reviewed in Sec. IV B). Finally we will discuss the general form of the two-point function for asymptotically large time and distance, that can be obtained from general CFT arguments.

\section{The gaussian model}

The content of this subsection has been already reported in Ref. [39], during the study of the time evolution of the entanglement entropy, that transforms like the two-point function of a primary field in a boundary gaussian theory [40]. We report it here for sake of completeness.

For a free boson the two-point function in the UHP is [35]

$$
\left\langle\Phi\left(z_{1}\right) \Phi\left(z_{2}\right)\right\rangle_{\mathrm{UHP}}=\left(\frac{z_{1 \overline{2}} z_{2 \overline{1}}}{z_{12} z_{\overline{1} \overline{2}} z_{1 \overline{1}} z_{2 \overline{2}}}\right)^{x}
$$

with $z_{i j}=\left|z_{i}-z_{j}\right|$ and $z_{\bar{k}}=\overline{z_{k}}$. Note that $\Phi$ is not the gaussian field $\theta(z)$, but its exponential $\Phi(z)=e^{i \theta(z)}($ see $\operatorname{Sec}$. IV A).

Under the conformal mapping (5) we obtain the two-point function on the strip at imaginary time $\tau$, at distance $r$ apart

$$
\begin{aligned}
\langle\Phi(r, \tau) \Phi(0, \tau)\rangle_{\text {strip }} & =\left|w^{\prime}\left(z_{1}\right)\right|^{-x}\left|w^{\prime}\left(z_{2}\right)\right|^{-x}\left\langle\Phi\left(z_{1}(w)\right)\left\langle\Phi\left(z_{2}(w)\right)\right\rangle_{\mathrm{UHP}}=\right. \\
& =\left[\left(\frac{\pi}{2 \tau_{0}}\right)^{2} \frac{\cosh \left(\pi r / 2 \tau_{0}\right)-\cos \left(\pi \tau / \tau_{0}\right)}{8 \sinh ^{2}\left(\pi r / 4 \tau_{0}\right) \sin ^{2}\left(\pi \tau / 2 \tau_{0}\right)}\right]^{x},
\end{aligned}
$$

that continued to real time $\tau=\tau_{0}+i t$ gives

$$
\langle\Phi(r, t) \Phi(0, t)\rangle=\left[\left(\frac{\pi}{2 \tau_{0}}\right)^{2} \frac{\cosh \left(\pi r / 2 \tau_{0}\right)+\cosh \left(\pi t / \tau_{0}\right)}{8 \sinh ^{2}\left(\pi r / 4 \tau_{0}\right) \cosh ^{2}\left(\pi t / 2 \tau_{0}\right)}\right]^{x} .
$$

In the case where $r / \tau_{0}$ and $t / \tau_{0}$ are large this simplifies to

$$
\langle\Phi(r, t) \Phi(0, t)\rangle=\left(\pi / 2 \tau_{0}\right)^{2 x}\left(\frac{e^{\pi r / 2 \tau_{0}}+e^{\pi t / \tau_{0}}}{e^{\pi r / 2 \tau_{0}} \cdot e^{\pi t / \tau_{0}}}\right)^{x} \propto\left\{\begin{array}{ll}
e^{-x \pi t / \tau_{0}} & \text { for } t<r / 2 \\
e^{-x \pi r / 2 \tau_{0}} & \text { for } t>r / 2
\end{array} .\right.
$$

i.e. the two point function at fixed $r$ decays exponentially in time up to $t^{*}=r / 2$ and then saturates to a value that depends exponentially on the separation.

In the case of fixed initial condition, with one-point function given by Eq. (8), the connected correlation function is

$$
\langle\Phi(r, t) \Phi(0, t)\rangle_{\text {conn }}=\langle\Phi(r, t) \Phi(0, t)\rangle-\langle\Phi(0, t)\rangle^{2} \propto \begin{cases}0 & \text { for } t<r / 2, \\ e^{-x \pi r / 2 \tau_{0}}-e^{-x \pi t / \tau_{0}} & \text { for } t>r / 2\end{cases}
$$

i.e. correlations start developing at $t^{*}=r / 2$ and, being $t \gg \tau_{0}$, at $t^{*}$ the connected two-point function almost immediately jumps to its asymptotic value. In the case of disordered initial conditions $\left(\psi_{0}(r)=0\right)$, connected and full correlation functions are equal.

\section{The Ising universality class}

For the Ising model the two-point function in the UHP is [35]

$$
\left\langle\Phi\left(z_{1}\right) \Phi\left(z_{2}\right)\right\rangle_{\mathrm{UHP}}=\left(\frac{z_{1 \overline{2}} z_{2 \overline{1}}}{z_{12} z_{\overline{1} \overline{2}} z_{1 \overline{1}} z_{2 \overline{2}}}\right)^{1 / 8} F(\eta)
$$

where $F(\eta)$ is given by

$$
F(\eta)=\frac{\sqrt{1+\eta^{1 / 2}} \pm \sqrt{1-\eta^{1 / 2}}}{\sqrt{2}}
$$


and $\eta$ is the four-point ratio

$$
\eta=\frac{z_{1 \overline{1}} z_{2 \overline{2}}}{z_{1 \overline{2}} z_{2 \overline{1}}}
$$

The sign \pm depends on the boundary conditions. + corresponds to fixed boundary conditions and and - to disordered ones.

The only difference with respect to the gaussian case is that we have also to map $F(\eta)$ according to the conformal transformation (5). After simple algebra we have

$$
\eta=\frac{2 \sin ^{2}\left(\pi \tau / 2 \tau_{0}\right)}{\cosh \left(\pi r / 2 \tau_{0}\right)-\cos \left(\pi \tau / \tau_{0}\right)}
$$

and so

$$
\begin{aligned}
& \langle\Phi(r, \tau) \Phi(0, \tau)\rangle_{\text {strip }}=\left[\left(\frac{\pi}{2 \tau_{0}}\right)^{2} \frac{\cosh \left(\pi r / 2 \tau_{0}\right)-\cos \left(\pi \tau / \tau_{0}\right)}{8 \sinh ^{2}\left(\pi r / 4 \tau_{0}\right) \sin ^{2}\left(\pi \tau / 2 \tau_{0}\right)}\right]^{1 / 8} \frac{1}{\sqrt{2}} \\
& \times\left[\sqrt{1+\frac{\sqrt{2} \sin \left(\pi \tau / 2 \tau_{0}\right)}{\sqrt{\cosh \left(\pi r / 2 \tau_{0}\right)-\cos \left(\pi \tau / \tau_{0}\right)}}} \pm \sqrt{1-\frac{\sqrt{2} \sin \left(\pi \tau / 2 \tau_{0}\right)}{\sqrt{\cosh \left(\pi r / 2 \tau_{0}\right)-\cos \left(\pi \tau / \tau_{0}\right)}}}\right] .
\end{aligned}
$$

Analytically continuing to real time $\tau=\tau_{0}+i t$ we obtain

$$
\begin{aligned}
& \langle\Phi(r, t) \Phi(0, t)\rangle=\left[\left(\frac{\pi}{2 \tau_{0}}\right)^{2} \frac{\cosh \left(\pi r / 2 \tau_{0}\right)+\cosh \left(\pi t / \tau_{0}\right)}{8 \sinh ^{2}\left(\pi r / 4 \tau_{0}\right) \cosh ^{2}\left(\pi t / 2 \tau_{0}\right)}\right]^{1 / 8} \frac{1}{\sqrt{2}} \\
& \times\left[\sqrt{1+\frac{\sqrt{2} \cosh \left(\pi t / 2 \tau_{0}\right)}{\sqrt{\cosh \left(\pi r / 2 \tau_{0}\right)+\cosh \left(\pi t / \tau_{0}\right)}}} \pm \sqrt{1-\frac{\sqrt{2} \cosh \left(\pi t / 2 \tau_{0}\right)}{\sqrt{\cosh \left(\pi r / 2 \tau_{0}\right)+\cosh \left(\pi t / \tau_{0}\right)}}}\right]
\end{aligned}
$$

that for $r / \tau_{0}$ and $t / \tau_{0}$ much larger than 1 simplifies to

$$
\left(\pi / 2 \tau_{0}\right)^{1 / 4} \frac{1}{\sqrt{2}}\left(\frac{e^{\pi r / 2 \tau_{0}}+e^{\pi t / \tau_{0}}}{e^{\pi r / 2 \tau_{0}} \cdot e^{\pi t / \tau_{0}}}\right)^{1 / 8}\left[\sqrt{1+\frac{e^{\pi t / 2 \tau_{0}}}{\sqrt{e^{\pi r / 2 \tau_{0}}+e^{\pi t / \tau_{0}}}}} \pm \sqrt{1-\frac{e^{\pi t / 2 \tau_{0}}}{\sqrt{e^{\pi r / 2 \tau_{0}}+e^{\pi t / \tau_{0}}}}}\right] .
$$

Note that the exponential terms in the square root are always $\ll 1$. Thus for fixed boundary condition we get the free boson result Eq. (12) with $x=1 / 8$. For the connected part we need to subtract $\langle\Phi(0, t)\rangle^{2}$ given by Eq. (8) with $A_{+}^{\Phi}=2^{1 / 4}$. We finally obtain

$$
\langle\Phi(r, t) \Phi(0, t)\rangle_{\mathrm{conn}}=\langle\Phi(r, t) \Phi(0, t)\rangle-\langle\Phi(0, t)\rangle^{2} \propto \begin{cases}0 & \text { for } t<r / 2, \\ e^{-\pi r / 16 \tau_{0}}-e^{-\pi t / 8 \tau_{0}} & \text { for } t>r / 2 .\end{cases}
$$

Thus, also for the Ising model with fixed boundary conditions, connected correlations start developing at $t=t^{*}=r / 2$. In the case of disordered initial condition, we have

$$
\langle\Phi(r, t) \Phi(0, t)\rangle \propto\left(\frac{e^{\pi r / 2 \tau_{0}}+e^{\pi t / \tau_{0}}}{e^{\pi r / 2 \tau_{0}} \cdot e^{\pi t / \tau_{0}}}\right)^{1 / 8} \frac{e^{\pi t / 2 \tau_{0}}}{\sqrt{e^{\pi r / 2 \tau_{0}}+e^{\pi t / \tau_{0}}}} \sim \begin{cases}e^{-\pi(r-3 / 2 t) / 4 \tau_{0}} & \text { for } t<r / 2, \\ e^{-\pi r / 16 \tau_{0}} & \text { for } t>r / 2,\end{cases}
$$

resulting in an exponential space dependence even for $t<r / 2$ (clearly in this case the connected correlation function equals the full one).

\section{The general two point-function}

From the results reported for the gaussian and Ising models, it is now relatively simple to understand the general properties of the time dependence of the two-point function in the very general case. The two-point function in the half-plane has the general form [35]

$$
\left\langle\Phi\left(z_{1}\right) \Phi\left(z_{2}\right)\right\rangle_{\mathrm{UHP}}=\left(\frac{z_{1 \overline{2}} z_{2 \overline{1}}}{z_{12} z_{\overline{1} \overline{2}} z_{1 \overline{1}} z_{2 \overline{2}}}\right)^{x} F(\eta)
$$



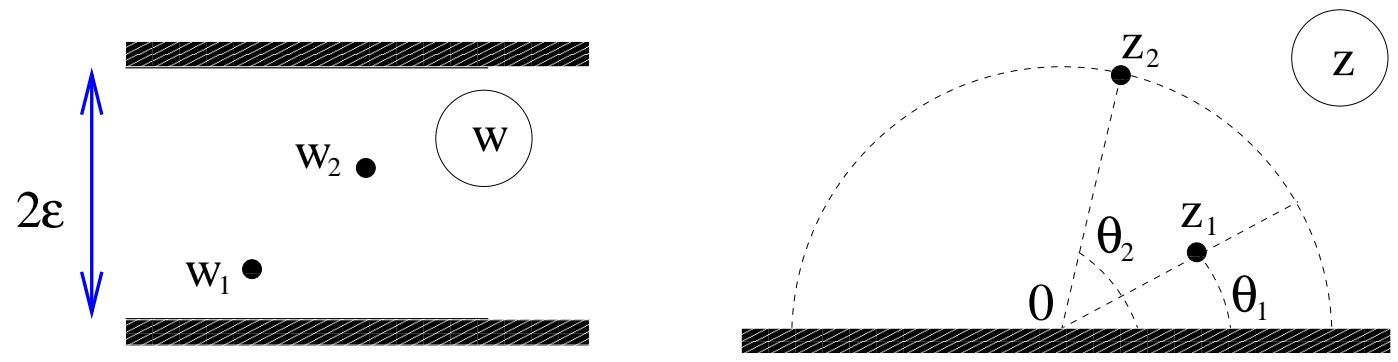

FIG. 2: Left: Space-time region for the correlation functions at different times. Right: Conformal mapping to the upper half-plane.

where the function $F(\eta)$ depends explicitly on the considered model. Under the conformal map to the strip we know that the first part of Eq. (23) transforms according to Eq. (10). Thus we need only to map $F(\eta)$ that, in the general case, is an unknown function. However, during the study of the Ising model we showed that the analytical continuation of $\eta$ for $t, r \gg \tau_{0}$ is

$$
\eta \sim \frac{e^{\pi t / \tau_{0}}}{e^{\pi r / 2 \tau_{0}}+e^{\pi t / \tau_{0}}}
$$

Thus for $t<r / 2$ we have $\eta \sim e^{\pi(t-r / 2) / \tau_{0}} \ll 1$ and in the opposite case $t>r / 2$ we have $\eta \sim 1$. As a consequence to have the asymptotic behavior of the two-point function we only need to know the behavior close to $\eta \sim 0$ (i.e. the behavior close to the surface) and for $\eta \sim 1$ (i.e. deep in the bulk). Fortunately they are both exactly known. Indeed when $\eta \sim 1$ the two points are deep in the bulk, meaning $F(1)=1$. Instead for $\eta \ll 1$, from the short-distance expansion, we have

$$
F(\eta) \simeq\left(A_{b}^{\Phi}\right)^{2} \eta^{x_{b}}
$$

where $x_{b}$ is the boundary scaling dimension of the leading boundary operator to which $\Phi$ couples and $A_{b}^{\Phi}$ is the bulk-boundary operator product expansion coefficient that equals the one introduced in Eq. (8) [see e.g. Ref. [37]].

All the previous observations and the explicit calculations of the previous sections lead for $t>r / 2$ to

$$
\langle\Phi(r, t) \Phi(0, t)\rangle \propto e^{-x \pi r / 2 \tau_{0}},
$$

while for $t<r / 2$ we get

$$
\langle\Phi(r, t) \Phi(0, t)\rangle \propto\left(A_{b}^{\Phi}\right)^{2} e^{-x \pi t / \tau_{0}} \times e^{\pi x_{b}(t-r / 2) / \tau_{0}} .
$$

Note that if $\langle\Phi\rangle \neq 0, x_{b}=0$ and the last factor is absent. The leading term is then just $\langle\Phi\rangle^{2}$. Thus the leading term in the connected two-point function vanishes for $t<r / 2$, and its first non-vanishing contributions is given by subleading terms either in $F$ or in the bulk-boundary short-distance expansion.

It is very interesting that we only have to know the behavior as $\eta \rightarrow 0$ and 1 to get the results we need for large $r$ and $t$. However, we stress that only a complete calculation (as those performed in the preceding sections) gives the full analytic structure of the CFT result needed to justify the analytical continuation from imaginary to real time. Moreover, the behavior within a distance $O\left(\tau_{0}\right)$ of the horizon $r=2 t$ depends on the detailed form of $F$.

\section{Correlations functions at different times}

Let us consider the case of the two-point function calculated at different real times $\langle\Phi(r, t) \Phi(0, s)\rangle$. This is again obtained by mapping the imaginary time strip to the UHP, but in this case the two points are $w_{1}=r+i \tau_{1}$ and $w_{2}=0+i \tau_{2}$, that, at the end of the calculation, must be analytically continued to $\tau_{1}=\tau_{0}+i t$ and $\tau_{2}=\tau_{0}+i s$. See Fig. 2 for a pictorial representation of the space-time domain in the strip and the resulting mapping to the UHP.

Let us start the discussion with the free boson. The distances appearing in Eq. (9) are

$$
\begin{array}{ll}
z_{12}^{2}=1+e^{\pi r / \tau_{0}}-2 e^{\pi r / 2 \tau_{0}} \cos \left(\theta_{1}-\theta_{2}\right), & z_{1 \overline{1}}=2 \sin \theta_{1}, \\
z_{1 \overline{2}}^{2}=1+e^{\pi r / \tau_{0}}-2 e^{\pi r / 2 \tau_{0}} \cos \left(\theta_{1}+\theta_{2}\right), & z_{2 \overline{2}}=2 e^{\pi r / 2 \tau_{0}} \sin \theta_{2},
\end{array}
$$


where $\theta_{i}=\pi \tau_{i} / 2 \tau_{0}$. Thus the correlation function on the strip is

$$
\begin{aligned}
& \left\langle\Phi\left(r, \tau_{1}\right) \Phi\left(0, \tau_{2}\right)\right\rangle_{\text {strip }}=\left|w^{\prime}\left(z_{1}\right)\right|^{-x}\left|w^{\prime}\left(z_{2}\right)\right|^{-x}\left\langle\Phi\left(z_{1}(w)\right)\left\langle\Phi\left(z_{2}(w)\right)\right\rangle_{\text {UHP }}=\right. \\
& =\left[\left(\frac{\pi}{2 \tau_{0}}\right)^{2} \frac{\cosh \left(\pi r / 2 \tau_{0}\right)-\cos \left(\pi\left(\tau_{1}+\tau_{2}\right) / 2 \tau_{0}\right)}{4 \sin \left(\pi \tau_{1} / 2 \tau_{0}\right) \sin \left(\pi \tau_{2} / 2 \tau_{0}\right)\left(\cosh \left(\pi r / 2 \tau_{0}\right)-\cos \left(\pi\left(\tau_{1}-\tau_{2}\right) / 2 \tau_{0}\right)\right)}\right]^{x},
\end{aligned}
$$

that for $\tau_{1}=\tau_{2}$ reduces to Eq. (10) as it should. Continuing to real times and considering $r, t, s,|t-s| \gg \tau_{0}$ we obtain

$$
\left(\frac{\pi}{2 \tau_{0}}\right)^{2 \Delta} \frac{e^{\frac{\Delta \pi r}{\tau_{0}}}+e^{\frac{\Delta \pi}{\tau_{0}}(t+s)}}{e^{\frac{\Delta \pi}{\tau_{0}}(t+s)}\left(e^{\frac{\Delta \pi r}{\tau_{0}}}+e^{\frac{\Delta \pi}{\tau_{0}}|t-s|}\right)}= \begin{cases}e^{-x \pi(t+s) / 4 \tau_{0}} & \text { for } r>t+s, \\ e^{-x \pi r / 4 \tau_{0}} & \text { for } t-s<r<t+s, \\ e^{-x \pi|t-s| / 4 \tau_{0}} & \text { for } r<|t-s| .\end{cases}
$$

Following the line sketched in the previous subsection, it is easy to generalize this result to the most general CFT. In the case of a theory with fixed initial conditions (i.e., $\langle\Phi\rangle \neq 0$ ) the asymptotic result is the same as before, with only the crossover points being affected by the precise expression for $F(\eta)$. Instead, in the case where $\langle\Phi\rangle=0$, the first case gains an additional factor $e^{-\pi x_{b}(t+s-r) / 4 \tau_{0}}$.

Note that the autocorrelation function (i.e. $r=0$ ) has only an exponential dependence on the time separation $t-s$ and does not exhibit aging in this regime.

\section{Evolution with boundaries}

We now consider the case of time evolution of a half-chain with some boundary condition at $r=0$. For simplicity we assume that the (conformal) boundary condition is of the same kind of the initial boundary condition (for example we fix all the spins at $t=0$ and at the boundary $r=0$ to point in the same direction). The space-time region we have to consider is depicted in Fig. 3. If different initial and boundary were considered, one needs to insert boundary conditions changing operators at the corners of the figure.

The $w$ plane is mapped into the UHP by

$$
z(w)=\sin \frac{\pi w}{2 \tau_{0}},
$$

with the corners at $\pm \tau_{0}$ mapped to \pm 1 . The mapping of $w_{1}$ is

$$
z_{1} \equiv z\left(w_{1}\right)=z\left(-\tau_{0}+\tau_{1}+i r\right)=-\cos \left(\pi \tau_{1} / 2 \tau_{0}\right) \cosh \left(\pi r / 2 \tau_{0}\right)+i \sin \left(\pi \tau_{1} / 2 \tau_{0}\right) \sinh \left(\pi r / 2 \tau_{0}\right) .
$$

In the $z$ plane the 1-point function is

$$
\left\langle\Phi\left(z_{1}\right)\right\rangle_{\mathrm{UHP}} \propto\left|\operatorname{Im} z_{1}\right|^{-x} \rightarrow\left[\sin \left(\pi \tau_{1} / 2 \tau_{0}\right) \sinh \left(\pi r / 2 \tau_{0}\right)\right]^{-x},
$$

and

$$
\left|w^{\prime}\left(z_{1}\right)\right|^{2}=\left(\frac{2 \tau_{0}}{\pi}\right)^{2} \frac{1}{\left|1-z^{2}\right|} \propto \frac{1}{\cosh \left(\pi r / \tau_{0}\right)-\cos \left(\pi \tau_{1} / \tau_{0}\right)} .
$$

Thus on the strip we have

$$
\left\langle\Phi\left(w_{1}\right)\right\rangle_{\text {strip }}=\left|w^{\prime}\left(z_{1}\right)\right|^{-x}\left\langle\Phi\left(w\left(z_{1}\right)\right)\right\rangle_{\mathrm{UHP}} \propto\left[\frac{\sin ^{2}\left(\pi \tau_{1} / 2 \tau_{0}\right) \sinh ^{2}\left(\pi r / 2 \tau_{0}\right)}{\cosh \left(\pi r / \tau_{0}\right)-\cos \left(\pi \tau_{1} / \tau_{0}\right)}\right]^{-x / 2},
$$

that continued to real time $\tau_{1}=i t$ is

$$
\langle\Phi(t, r)\rangle \propto\left[\frac{\cosh \left(\pi t / \tau_{0}\right)+\cosh \left(\pi r / \tau_{0}\right)}{\cosh \left(\pi t / 2 \tau_{0}\right)^{2} \sinh ^{2}\left(\pi r / 2 \tau_{0}\right)}\right]^{x / 2}
$$

and for $t, r \gg \tau_{0}$ simplifies to

$$
\left\langle\Phi(t, r) \propto\left[\frac{e^{\pi r / \tau_{0}}+e^{\pi t / \tau_{0}}}{e^{\pi r / \tau_{0}} \cdot e^{\pi t / \tau_{0}}}\right]^{x / 2}= \begin{cases}e^{-\pi x t / 2 \tau_{0}} & \text { for } t<r \\ e^{-\pi x r / 2 \tau_{0}} & \text { for } t>r .\end{cases}\right.
$$

Note that in this case the characteristic time is $t^{*}=r$ and not $r / 2$. This explains also why the entanglement entropy of a semi-infinite chain with free boundary condition at $x=0$, has characteristic time $t^{*}=r$ as firstly noted in Ref. $[41]$. 

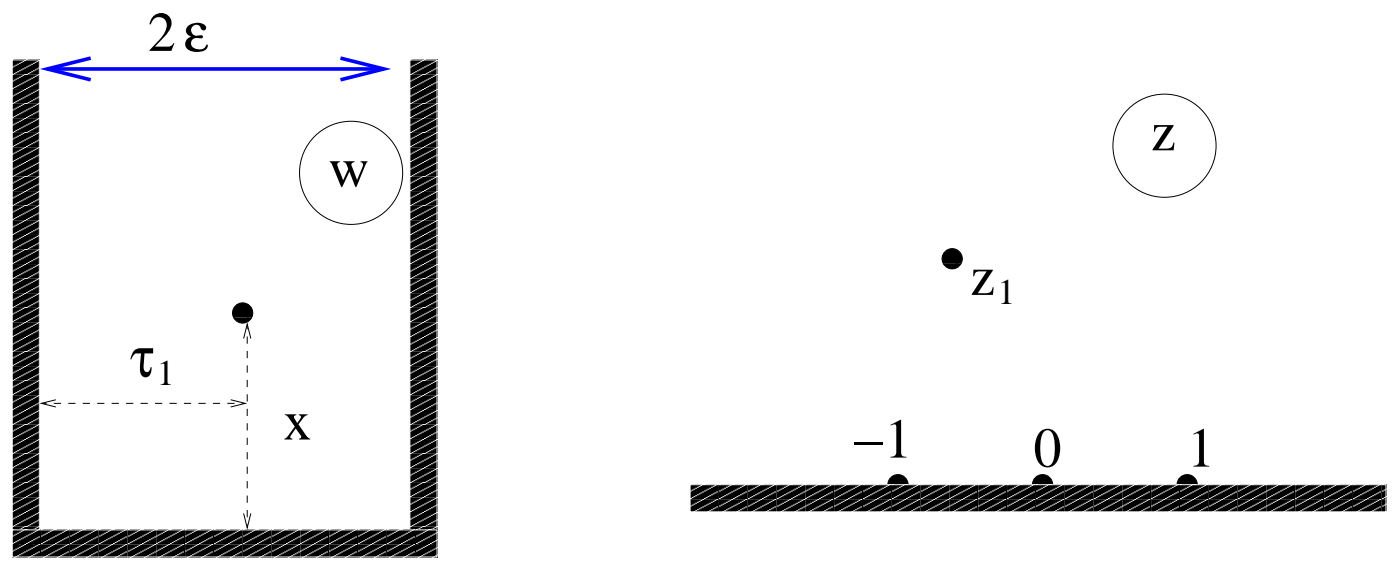

FIG. 3: Left: Space-time region for the one-point function in a boundary (at $r=0$ ) geometry. Note $w=\tau+i r$. Right: Conformal mapping to the upper-half plane, c.f. Eq. (31).

\section{E. Discussion and interpretation of the CFT results}

All the correlation functions calculated so far display two very general features: first there is a sharp horizon (or light-cone) effect at $t=t^{*}=r / 2$ (or $r$ ) resulting in a behavior before and after $t^{*}$ completely different; second the asymptotic long-time correlation functions are the same as those at finite temperature $\beta_{\mathrm{eff}}=4 \tau_{0}$. The light-cone effect is a very general phenomenon and and will be discussed in section VI. In the following sections we will point out that also the "effective temperature" is a general phenomenon, but it has some specific CFT features that are worthy of comment.

In fact, it is very easy to understand the technical reason why we find an effective temperature despite the fact that we are studying a pure state at $T=0$. The finite temperature correlations can be calculated by studying the field theory on a cylinder of circumference $\beta=1 / T$. In CFT a cylinder can be obtained by mapping the complex plane with the logarithmic transformation $\beta /(2 \pi) \log z$. The form for the two-point function in the slab depends in general on the function $F(\eta)$-cf. Eq. (15)- but when we analytically continue and take the limit of large real time, we find that effectively the points are far from the boundary, i.e. at $\eta=1$. Thus we get the same result as we would get if we conformally transformed from the full plane to a cylinder, and from Eq. (5) the effective temperature is $\beta_{\text {eff }}=4 \tau_{0}$. A similar argument can be worked out for the multi-point functions as well.

An addtional comment concerns what we expect for correlation functions of general operators, not only primary. We have clearly seen that the local energy density does not relax, of course, and this is consistent with its not being primary. But, we also know that at finite temperature $\left\langle T_{t t}\right\rangle_{\beta}=\pi c / 6 \beta^{2}$, that is perfectly compatible with the previous result with $\beta_{\text {eff }}=4 \tau_{0}$. Furthermore, for large real times, the two-point function of $T_{t t}-\langle T\rangle_{t t}$ does behave as though it was at finite temperature. This means, in particular, that the energy fluctuations in a large but finite volume (the specific heat) are the same as those at finite temperature. Thus one is tempted to extend this finite temperature interpretation to non-primary operators, and indeed it is the case. In fact, in the argument of above for the equivalence of the long-time correlations and finite $T$, there are essentially three steps. First, we need to write down the form of the correlation function in the half-plane, but this only depends on special conformal transformation and so is valid for any quasi-primary operator like $T_{\mu \nu}$. Second we have to transform from the slab to the half-plane: for non-primary operators this can have some anomalous term. Finally we need to compare the large limit of this to what one would get transforming directly from the cylinder to the full plane. However, the two conformal transformations we are comparing are both logarithmic (in one case $2 \tau_{0} / \pi \log z$, in the other case $\beta / 2 \pi \log z$ ), so the anomalous terms should be the same. Thus the two correlations functions are the same. This argument works for all quasi-primary operators. Then, since we can get all the non-quasiprimaries by considering successive operator product expansions with the stress tensor, it also works for all operators.

\section{EXACT REAL-TIME DYNAMICS IN SIMPLE INTEGRABLE CHAINS}

The results of the previous section rely on the technical assumption that the leading asymptotic behavior given by CFT, which applies to the euclidean region (large imaginary times), may simply be analytically continued to find the behavior at large real time. While such procedures have been shown to give the correct behavior for the 
time-dependent correlations in equilibrium, it is important to check them in specific solvable cases for non-equilibrium evolution.

Thus, as a complement to the CFT calculations, in this section we consider the real-time evolution of two simple analytically tractable models. We solve the dynamics of a chain of coupled harmonic oscillators and we review and re-analyze some known results for the Ising-XY chain in a transverse magnetic field. Beyond providing examples of the CFT results (with central charge $c=1$ and $1 / 2$ respectively) in the critical case, these models allow us to take into account the effects of a finite mass-gap and of the lattice.

\section{A. The chain of harmonic oscillators}

The simplest model with an exactly solvable non-equilibrium dynamics is surely a chain of coupled harmonic oscillators with hamiltonian

$$
H=\frac{1}{2} \sum_{r}\left[\pi_{r}^{2}+m^{2} \phi_{r}^{2}+\sum_{j} \omega_{j}^{2}\left(\phi_{r+j}-\phi_{r}\right)^{2}\right] .
$$

We introduce a coupling more general than simple nearest-neighbor hopping so as to allow for a general dispersion relation below. For simplicity we also assume periodic boundary conditions. $\varphi_{n}$ and $\pi_{n}$ are the position and the momentum operators of the $n$-th oscillator, with equal time commuting relations

$$
\left[\varphi_{m}, \pi_{n}\right]=i \delta_{n m} \quad\left[\varphi_{n}, \varphi_{m}\right]=\left[\pi_{n}, \pi_{m}\right]=0 .
$$

The hamiltonian can be written in diagonal form $H(m)=\sum_{k} \Omega_{k} A_{k}^{\dagger} A_{k}$ with modes

$$
\begin{aligned}
A_{k} & =\frac{1}{\sqrt{2 \Omega_{k}}}\left(\Omega_{k} \varphi_{k}+i \pi_{k}\right), \\
A_{k}^{\dagger} & =\frac{1}{\sqrt{2 \Omega_{k}}}\left(\Omega_{k} \varphi_{-k}-i \pi_{-k}\right), \\
\Omega_{k}^{2} & =m^{2}+2 \sum_{j} \omega_{j}(1-\cos (2 \pi k j / N)) .
\end{aligned}
$$

Note that we use the same symbols for the operators and their Fourier transforms $\left(\varphi_{k}=1 / \sqrt{N} \sum_{n=0}^{N-1} e^{2 \pi i k n / N} \varphi_{n}\right.$ and analogously for $\pi_{k}$ ).

We consider the scenario in which the system is prepared in a state $\left|\psi_{0}\right\rangle$, that is ground-state of $H\left(m_{0}\right)$, and at the time $t=0$ the mass is quenched to a different value $m \neq m_{0}$. We use the notation $\Omega_{0 k}$ for the dispersion relation for $t<0$ and the $\Omega_{k}$ for the one for $t>0$.

Since $\left\langle\psi_{0}\left|\varphi_{n}\right| \psi_{0}\right\rangle=0$, the expectation value of the field $\varphi_{n}$ vanishes at any time. This example in fact corresponds to the quench from the disordered phase in the language of the previous section. Thus we concentrate our attention on the two-point function

$$
\left\langle\psi(t)\left|\varphi_{n} \varphi_{0}\right| \psi(t)\right\rangle=\left\langle\psi_{0}\left|\varphi_{n}^{H}(t) \varphi_{0}^{H}(t)\right| \psi_{0}\right\rangle
$$

where we introduced the operator in the Heisenberg picture $\varphi_{n}^{H}(t)$, whose time evolution is given by

$$
\varphi_{n}^{H}(t)=\sum_{k=0}^{N-1} \sqrt{\frac{2}{N \Omega_{k}}}\left(e^{i\left(p_{k} n-\Omega_{k} t\right)} A_{k}+e^{-i\left(p_{k} n-\Omega_{k} t\right)} A_{k}^{\dagger}\right),
$$

where $p_{k}=2 \pi k / N$. Accordingly, the product of the two fields is

$$
\varphi_{n}^{H}(t) \varphi_{0}^{H}(t)=\frac{2}{N} \sum_{k, k^{\prime}} \frac{1}{\sqrt{\Omega_{k} \Omega_{k^{\prime}}}}\left(e^{i\left(p_{k} n-\Omega_{k} t\right)} A_{k}+e^{-i\left(p_{k} n-\Omega_{k} t\right)} A_{k}^{\dagger}\right)\left(e^{-i \Omega_{k^{\prime}} t} A_{k^{\prime}}+e^{i \Omega_{k^{\prime}} t} A_{k^{\prime}}^{\dagger}\right) .
$$

In order to have the time dependent two-point function we need the expectation values of the bilinear combinations of $A$ 's on the initial state, that is annihilated by the $A_{0 k}$. Thus it is enough to write $A$ 's as functions of $A_{0}$ 's, i.e.

$$
\begin{aligned}
& A_{k}=\frac{1}{2}\left[A_{0 k}\left(\sqrt{\frac{\Omega_{k}}{\Omega_{0 k}}}+\sqrt{\frac{\Omega_{0 k}}{\Omega_{k}}}\right)+A_{0-k}^{\dagger}\left(\sqrt{\frac{\Omega_{k}}{\Omega_{0 k}}}-\sqrt{\frac{\Omega_{0 k}}{\Omega_{k}}}\right)\right] \equiv c_{k} A_{0 k}+d_{k} A_{0-k}^{\dagger}, \\
& A_{k}^{\dagger}=\frac{1}{2}\left[A_{0 k}^{\dagger}\left(\sqrt{\frac{\Omega_{k}}{\Omega_{0 k}}}+\sqrt{\frac{\Omega_{0 k}}{\Omega_{k}}}\right)+A_{0-k}\left(\sqrt{\frac{\Omega_{k}}{\Omega_{0 k}}}-\sqrt{\frac{\Omega_{0 k}}{\Omega_{k}}}\right)\right] \equiv c_{k} A_{0 k}^{\dagger}+d_{k} A_{0-k},
\end{aligned}
$$


leading to (we understand $\langle\cdot\rangle=\left\langle\psi_{0}|\cdot| \psi_{0}\right\rangle$ )

$$
\begin{aligned}
\left\langle A_{k} A_{k^{\prime}}\right\rangle & =\left\langle\left(c_{k} A_{0 k}+d_{k} A_{0-k}^{\dagger}\right)\left(c_{k^{\prime}} A_{0 k^{\prime}}+d_{k^{\prime}} A_{0-k^{\prime}}^{\dagger}\right)\right\rangle=c_{k} d_{k^{\prime}}\left\langle A_{0 k} A_{0-k^{\prime}}^{\dagger}\right\rangle=c_{k} d_{k} \delta_{k,-k^{\prime}}, \\
\left\langle A_{k} A_{k^{\prime}}^{\dagger}\right\rangle & =\left\langle\left(c_{k} A_{0 k}+d_{k} A_{0-k}^{\dagger}\right)\left(c_{k^{\prime}} A_{0 k^{\prime}}^{\dagger}+d_{k^{\prime}} A_{0-k^{\prime}}\right)\right\rangle=c_{k} c_{k^{\prime}}\left\langle A_{0 k} A_{0 k^{\prime}}^{\dagger}\right\rangle=c_{k}^{2} \delta_{k, k^{\prime}}, \\
\left\langle A_{k}^{\dagger} A_{k^{\prime}}\right\rangle & =\left\langle\left(c_{k} A_{0 k}^{\dagger}+d_{k} A_{0-k}\right)\left(c_{k^{\prime}} A_{0 k^{\prime}}+d_{k^{\prime}} A_{0-k^{\prime}}^{\dagger}\right)\right\rangle=d_{k} d_{k^{\prime}}\left\langle A_{0-k} A_{0-k^{\prime}}^{\dagger}\right\rangle=d_{k}^{2} \delta_{k, k^{\prime}}, \\
\left\langle A_{k}^{\dagger} A_{k^{\prime}}^{\dagger}\right\rangle & =\left\langle\left(c_{k} A_{0 k}^{\dagger}+d_{k} A_{0-k}\right)\left(c_{k^{\prime}} A_{0 k^{\prime}}^{\dagger}+d_{k^{\prime}} A_{0-k^{\prime}}\right)\right\rangle=d_{k} c_{k^{\prime}}\left\langle A_{0-k} A_{0 k^{\prime}}^{\dagger}\right\rangle=c_{k} d_{k} \delta_{k,-k^{\prime}} .
\end{aligned}
$$

Finally we arrive at

$$
\left\langle\varphi_{n}^{H}(t) \varphi_{0}^{H}(t)\right\rangle=\frac{2}{N a} \sum_{k} \frac{1}{\Omega_{k}}\left[c_{k} d_{k} e^{i\left(p_{k} n-2 \Omega_{k} t\right)}+c_{k}^{2} e^{i p_{k} n}+d_{k}^{2} e^{-i p_{k} n}+c_{k} d_{k} e^{-i\left(p_{k} n-2 \Omega_{k} t\right)}\right],
$$

which, in the thermodynamic limit $(N \rightarrow \infty)$, may be written as

$$
\left\langle\varphi_{r}^{H}(t) \varphi_{0}^{H}(t)\right\rangle-\left\langle\varphi_{r}^{H}(0) \varphi_{0}^{H}(0)\right\rangle=\int_{\mathrm{BZ}} \frac{d k}{(2 \pi)} e^{i k r} \frac{\left(\Omega_{0 k}^{2}-\Omega_{k}^{2}\right)\left(1-\cos \left(2 \Omega_{k} t\right)\right)}{\Omega_{k}^{2} \Omega_{0 k}},
$$

where the integral is on th first Brillouin zone $|k|<\pi / a$. Note that for $t=0$ and for $m=m_{0}$ this two-point function reduces to the static one, as it should. This result can also be found by integrating the Heisenberg equations of motion for each mode. For future reference it is also useful to write down explicitly the Fourier transform known as momentum distribution function

$$
\rho(k)=\frac{\left(\Omega_{0 k}^{2}+\Omega_{k}^{2}\right)-\left(\Omega_{0 k}^{2}-\Omega_{k}^{2}\right) \cos \left(2 \Omega_{k} t\right)}{\Omega_{k}^{2} \Omega_{0 k}} .
$$

Note that when considering correlation functions in momentum space, a long-time limit does not exist and we need to take the time average, in contrast to what happens in real space.

\section{The continuum limit}

In Eq. (52) everything is completely general and applies to any chain with finite lattice spacing. Let us know discuss the continuum limit that is achieved by sending $N \rightarrow \infty$ in such a way that $(1 / N) \sum_{k} \rightarrow \int_{-\infty}^{\infty} d p /(2 \pi)$, $p_{n} \rightarrow p$, and $\Omega_{k}^{2} \rightarrow \Omega_{p}^{2}=m^{2}+p^{2}$

In this limit the correlation function becomes $\left(\Delta m^{2}=m_{0}^{2}-m^{2}\right)$

$$
G(r, t) \equiv\left\langle\varphi_{r}^{H}(t) \varphi_{0}^{H}(t)\right\rangle=\int_{-\infty}^{\infty} \frac{d p}{2 \pi} e^{i p r} \frac{-\Delta m^{2} \cos \left(2 \sqrt{p^{2}+m^{2}} t\right)+m^{2}+m_{0}^{2}+2 p^{2}}{\left(m^{2}+p^{2}\right) \sqrt{m_{0}^{2}+p^{2}}} .
$$

Since a closed form for such integral is quite difficult to write down in the most general case, we will only consider some particular cases.

Let us first consider the conformal evolution $(m=0)$ from an initial state with a very large mass $m_{0} \rightarrow \infty$. This should reproduce the CFT result previous section for all the time $t$, since the correlations of the initial state shrink to a point. We have

$$
G(r, t)=m_{0} \int_{-\infty}^{\infty} \frac{d p}{2 \pi} e^{i p r} \frac{1-\cos (2 p t)}{p^{2}}=m_{0} \begin{cases}0 & \text { for } t<r / 2 \\ \frac{1}{2}(2 t-r) & \text { for } t>r / 2\end{cases}
$$

To compare such result with the conformal result given by Eq. (12), we have to keep in mind that the primary field is not the gaussian one $\varphi^{H}(r, t)$, but its imaginary exponential. Thus we need $\left\langle e^{i q \varphi^{H}(r, t)} e^{-i q \varphi^{H}(0, t)}\right\rangle$, with an arbitrary $q$. Despite of the apparent complexity of such correlator, it is very simple to obtain it using the standard property of gaussian integrals

$$
\left\langle e^{i q \varphi^{H}(r, t)} e^{-i q \varphi^{H}(0, t)}\right\rangle=e^{-q^{2}\left\langle\left(\varphi^{H}(r, t)-\varphi^{H}(0, t)\right)^{2}\right\rangle / 2}=e^{q^{2}(G(r, t)-G(0, t))}
$$

leading to

$$
\left\langle e^{i q \varphi^{H}(r, t)} e^{-i q \varphi^{H}(0, t)}\right\rangle= \begin{cases}e^{-q^{2} m_{0} t} & \text { for } t<r / 2 \\ e^{-q^{2} m_{0} r / 2} & \text { for } t>r / 2\end{cases}
$$



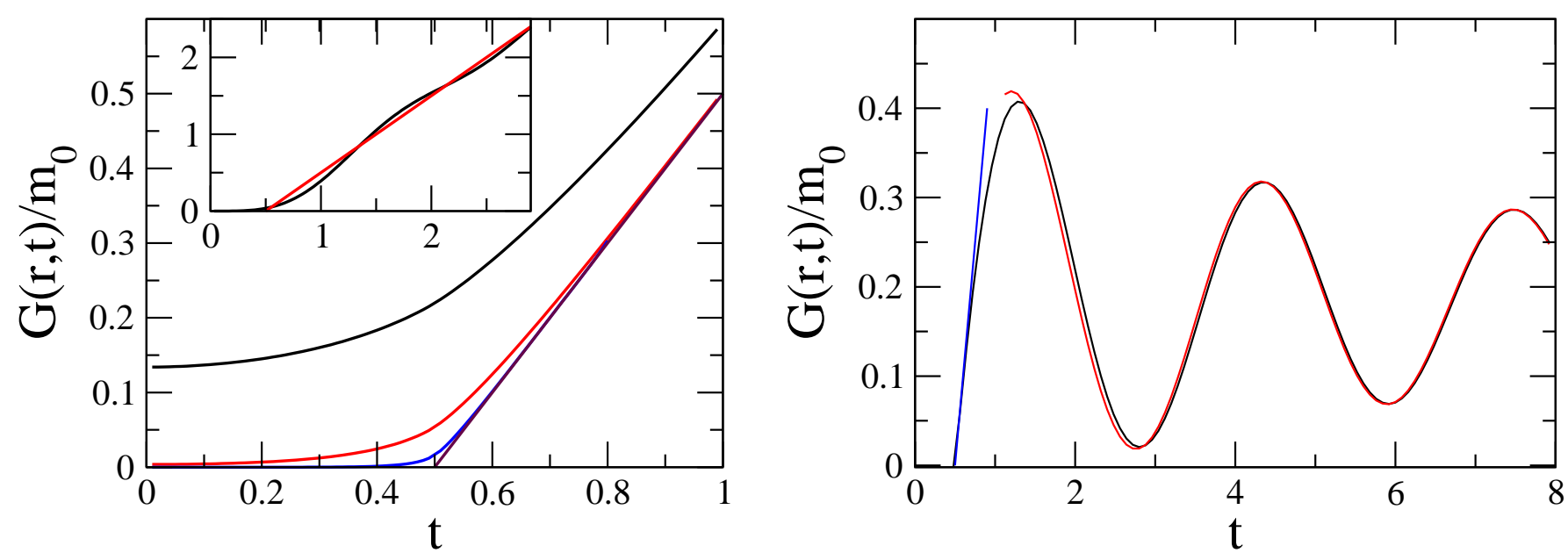

FIG. 4: Left: $G(r, t) / m_{0}$ given by Eq. (60) as function of $t$, at fixed $r=1$. Three different values of $m_{0}=10,3,1$ (from the bottom to the top) are shown. Inset: Lattice effects showing the cos $4 t$ oscillations on top of the continuum result. Right: $G(r, t) / m_{0}$ given by the numerical integral of Eq. (62) as function of $t$, at fixed $m, r=1$. It is compared with the asymptotic behavior for $0<2 t-r \ll m^{-1}$ and for $t \gg r$.

that is exactly the same of Eq. (12) with $x_{\Phi} \propto q^{2}$ and $\tau_{0} \propto m_{0}^{-1}$, confirming that $\tau_{0}$ is just proportional to the correlation in the initial state, as its interpretation in terms of the extrapolation length suggests.

The case $m=0$ and $m_{0}$ finite corresponds to a conformal evolution from a generic state with correlations proportional to $m_{0}^{-1}$. Thus we expect the CFT result Eq. (12) to be true for asymptotic large times and separations.

From Eq. (55), the two-point function of the gaussian field is

$$
G(r, t)=\int_{-\infty}^{\infty} \frac{d p}{2 \pi} e^{i p r} \frac{-m_{0}^{2} \cos (2 p t)+m_{0}^{2}+2 p^{2}}{p^{2} \sqrt{m_{0}^{2}+p^{2}}},
$$

that can be written as

$$
2 \pi G(r, t)=2 K_{0}\left(m_{0} r\right)+f\left(m_{0} r\right)-\frac{f\left(m_{0}(r-2 t)\right)+f\left(m_{0}(r+2 t)\right)}{2},
$$

where $K_{0}(y)$ is the modified Bessel function and

$$
f(y)=1+\frac{1}{2} G_{13}^{21}\left(\frac{y^{2}}{4} \mid \begin{array}{ccc}
3 / 2 & \\
0 & 1 & 1 / 2
\end{array}\right),
$$

with $G_{13}^{21}$ the Meijer $G$-function (see e.g. [42]). Note that $f(x)$ is characterized by $f^{\prime \prime}(x)=-K_{0}(x)$ and $f(0)=f^{\prime}(0)=$ 0 . In the limit $m_{0} \rightarrow \infty$, it is easy to show that $G(r, t)$ reduces to the previous result. In the left panel of Fig. 4 , we report $G(r, t)$ as function of $t$ at fixed $r=1$ for $m_{0}=10,3,1$. It is evident that a finite $m_{0}$ results in smoothing the curve close to $t=r / 2$ and giving an offset in zero. Both the effects are more pronounced as $m_{0}$ decreases. For large $t$, independently on $m_{0} \neq 0$, we have $G(r, t)=t+O\left(t^{0}\right)$, confirming that the CFT result is correct for asymptotic large times.

The case with arbitrary $m$ and $m_{0}$ is quite cumbersome to be worked out analytically and not really illuminating. For this reason we concentrate here on the massive evolution from a state with $m_{0} \rightarrow \infty$. In this case the correlation function reads

$$
G(r, t)=m_{0} \int_{-\infty}^{\infty} \frac{d p}{2 \pi} e^{i p r} \frac{1-\cos \left(2 \sqrt{p^{2}+m^{2}} t\right)}{\left(m^{2}+p^{2}\right)} .
$$

In the limit $t \rightarrow \infty$ the cosine term averages to zero, giving

$$
G(r, t=\infty)=m_{0} \int_{-\infty}^{\infty} \frac{d p}{2 \pi} \frac{e^{i p r}}{\left(m^{2}+p^{2}\right)}=\frac{m_{0}}{m} \frac{e^{-m r}}{2} .
$$

To understand the time dependence, let us first note that, despite the presence of a square-root, the integrand of Eq. (62) is analytic in $p$, since the square-root is the argument of the even cosine function. Thus we can make the 
integral in the complex plane and use the Cauchy theorem. As long as $r>2 t$, the behavior for $p \rightarrow i \infty$ is dominated by $e^{i p r}$ and we can safely close the contour path in the upper half-plane where the residue at $p=i m$ is zero. As a consequence $G(r, t<r / 2)=0$.

For $t>r / 2$, the integral is more difficult, since we can not close the contour in the upper half-plane, because the cosine is "larger" than $e^{i p r}$ for $p \rightarrow i \infty$. However, the approach to the asymptotic value is easily worked out. In fact, for $t \gg r$, the term $e^{i p r}$ in the integral (62) can be approximated with 1, since it is slowly oscillating. Thus we have

$$
G(r, t \gg r)-G(r, t=\infty) \simeq-m_{0} \int_{-\infty}^{\infty} \frac{d p}{2 \pi} \frac{\cos \left(2 \sqrt{p^{2}+m^{2}} t\right)}{\left(m^{2}+p^{2}\right)}=-m_{0} f_{m}(t),
$$

where $f_{m}(t)=1 /(2 m)-t_{1} F_{2}\left(1 / 2 ; 1,3 / 2 ;-m^{2} t^{2}\right)$, that satisfies $f_{m}^{\prime}(t)=-J_{0}(2 m t)$ and $f_{m}(0)=1 /(2 m)$. Note that $f_{m}(t)$ is just the result for $r=0$. In the complementary region $0<2 t-r \ll m^{-1}$, the integral in Eq. (62) is dominated by the modes with $p \gg m$, and so it can be described by the conformal result.

In the right panel of Fig. 4, we plot the time-dependence of $G(r, t)$ at fixed $r$ as obtained by numerically integrating Eq. (62) for $m=r=1$. The plot shows that the conformal result $t-r / 2$ describes the behavior close to $t=r / 2$, while for larger times the asymptotic expression gives an excellent approximation.

We finally note that $G(r, t)$ at fixed $t$ displays spatial oscillations (for $r<2 t$, else it vanishes), as it can be simply realized by a stationary phase argument.

\section{Lattice effects}

Another advantage of this simple model is that the effects of the lattice can be easily understood. In fact, by a stationary phase argument, the dominant contribution to (53) in the limits of large $t$ and $r$ comes from where, the group velocity $v_{k} \equiv \Omega_{k}^{\prime}=r / 2 t$, independently of the explicit form of $\Omega_{k}$. Consequently, the two-point function of a gapless model with dispersion relation $\Omega_{k}=2 \sin k / 2$ differs from the continuum limit previously derived for $t>r / 2 v_{m}$, where $v_{m}$ is the maximum group velocity. In particular $G(r, t)$ receives a contribution from the slowest mode $\left(k=\pi\right.$ with $\left.v_{\pi}=0\right)$ whose effect is to add to Eq. (56) fast oscillations going as $\cos \left(2 \Omega_{\pi} t\right)=\cos 4 t$. The resulting $G(r, t)$ is plotted in the inset of Fig. 4 in the gapless case.

Thus, lattice effects play a more important role in the cases where the asymptotic result vanishes. A typical example is the energy density that in this model is proportional to $\left(\varphi_{r+1}-\varphi_{r}\right)^{2}$. The continuum calculation would just give zero (the mean-energy is conserved), but the approach to this value is governed by lattice effects and is dominated by the smallest group velocity that comes from the zone boundary at $|k|=\pi$. In fact, just with a trivial calculation we have

$$
\begin{aligned}
\left\langle\left(\varphi_{r+1}-\varphi_{r}\right)^{2}\right\rangle & =2\left(\left\langle\varphi_{0}^{2}\right\rangle-\left\langle\varphi_{0} \varphi_{1}\right\rangle\right)=\int \frac{d k}{2 \pi}(1-\cos k) \frac{\left(1-\cos 2 \Omega_{k} t\right)}{2 \sin ^{2} k / 2}= \\
& =\int \frac{d k}{2 \pi}\left(1-\cos 2 \Omega_{k} t\right) \propto J_{0}(4 t) \sim t^{-1 / 2} \cos 4 t,
\end{aligned}
$$

that displays the typical $\cos 4 t$ from the zone boundary at $|k|=\pi$.

The lattice dispersion relations are sensitive to the microscopical details of the model and consequently quantities like the energy-density show a dependence on this. For example, a lattice massless fermion has dispersion relation $\Omega_{k}=2 \sin k / 2$ and energy density given by $\sin ^{2} k$. In this case the time evolution of the energy density is

$$
\int \frac{d k}{2 \pi} \sin ^{2} k \frac{\left(1-\cos 2 \Omega_{k} t\right)}{2 \sin ^{2} k / 2},
$$

that for large times goes like $t^{-3 / 2} \cos 4 t$, resulting in a different power law compared to before.

If the system is quenched to a gapped $H$ with $\Omega_{k}^{2}=m^{2}+2(1-\cos k)$ the maximum group velocity corresponds to a non-zero wave number. This gives rise to spatial oscillations in the correlation function (this is true also in the continuum limit as already discussed in the previous subsection). 


\section{B. The Ising-XY chain in a transverse magnetic field}

The most studied one dimensional quantum spin model is the so-called XY chain in a transverse field, defined by the hamiltonian

$$
H=\sum_{j=1}^{N}\left[\frac{(1+\gamma)}{2} \sigma_{j}^{x} \sigma_{j+1}^{x}+\frac{(1-\gamma)}{2} \sigma_{j}^{y} \sigma_{j+1}^{y}-h \sigma_{z}\right],
$$

where $\sigma_{i}^{x, y, z}$ are the Pauli matrices, $\gamma$ is called anisotropy parameter, and $h$ is the applied external transverse field. It is well known that for any $\gamma \neq 0$ the model undergoes a phase transition at $h=1$, that is in the universality class of the Ising model (defined by $\gamma=1$ ). For simplicity we will just consider the Ising case, other values of $\gamma \neq 0$ being equivalent.

We consider the non-equilibrium unitary dynamics that follows from a quench of the magnetic field at $t=0$ from $h_{0}$ to $h_{1} \neq h_{0}$. Earlier works on this subject by Barouch et al. date back to seventies $[7,8]$. Exploiting the mapping of this model onto a free fermion, the time evolution of the transverse magnetization (that is not the order parameter) was obtained exactly [7]:

$$
m_{z}(t)=\frac{1}{\pi} \int_{0}^{\pi} d k \frac{\left(h_{0}-h_{1}\right) \sin ^{2} k \cos \left(2 \epsilon_{1} t\right)-\left(\cos k-h_{1}\right)\left[\left(\cos k-h_{0}\right)\left(\cos k-h_{1}\right)+\sin ^{2} k\right]}{\epsilon_{0} \epsilon_{1}^{2}},
$$

where $\epsilon_{i}=\epsilon\left(h_{i}\right)$ with $\epsilon(h)=\sqrt{\sin ^{2} k+(h-\cos k)^{2}}$. In Ref. [7] it was shown that (for non-exceptional parameters) the approach to the asymptotic value for $t \rightarrow \infty$ is of the form $t^{-3 / 2} \cos 4 t$. This is simply shown in the case of $h_{0}=\infty$ and $h_{1}=1$, when the integral simplifies and we obtain (see also [9])

$$
m_{z}(t)=\frac{1}{2}+\frac{1}{\pi} \int_{0}^{\pi} d k \frac{\sin ^{2} k \cos \left(2 \epsilon_{1} t\right)}{4 \sin ^{2} k / 2}=\frac{1}{2}+\frac{J_{1}(4 t)}{4 t},
$$

where $J_{1}$ is the Bessel function of the first kind whose asymptotic expansion for large argument is $J_{1}(x) \sim$ $\sqrt{2 / \pi x} \cos (x+\pi / 4)$.

To understand this result we should keep in mind that the transverse magnetization is not the order parameter: it corresponds to the product of two "disorder parameters" at neighbor sites on the dual lattice. Thus it must have the symmetry of an energy operator, for which CFT just predicts a constant asymptotic result. The power law term $t^{-3 / 2} \cos (4 t)$ is just the lattice correction to the asymptotic. This correction clearly shows the fermionic nature of the model on the basis of what discussed in the previous section.

The asymptotic result of the two-point function for $t \rightarrow \infty$ has been studied in Ref. [11]. Calling $G_{n}=$ $\langle\Phi(n, \infty) \Phi(0, \infty)\rangle$, in the case $h_{0}=\infty$ it has been found

$$
G_{n}= \begin{cases}\frac{1}{2^{n} h_{1}^{n}}, & \text { for } h_{1} \geq 1, \\ \frac{1}{2^{n}} \cos \left[n \arccos \left(h_{1}\right)\right], & \text { for } h_{1} \leq 1\end{cases}
$$

instead for $h_{0}=0$

$$
G_{n}= \begin{cases}\frac{1}{2^{n}}, & \text { for } h_{1} \geq 1, \\ \frac{h_{1}^{n+1}}{2^{n}} \cosh \left[(n+1) \ln \left(\frac{1+\sqrt{1-h_{1}^{2}}}{h_{1}}\right)\right], & \text { for } h_{1} \leq 1,\end{cases}
$$

that for large $n$ decay exponentially with $n$. It has been shown that $G_{n}$ is decaying exponentially with $n$ for general $h_{0}$ and $h_{1}$ [11], although closed forms are not available. The exponential decay is the prediction of CFT that (maybe surprisingly) applies even far from the critical point $h_{1}=1$.

The time dependence of two-point function has been studied in Ref. [9] by means of exact diagonalization of the model with open boundary condition at the two ends $r=0, L$. The results of interest for this paper are

- The connected two-point function of $\sigma_{z}$ in the thermodynamic limit and at the critical point is

$$
\left\langle\sigma_{r}^{z}(t) \sigma_{0}^{z}(t)\right\rangle_{c}=\left[\frac{r}{2 t} J_{2 r}(4 t)\right]^{2}-\frac{r^{2}-1}{4 t^{2}} J_{2 r+1}(4 t) J_{2 r-1}(4 t),
$$

which is valid both for $h_{0}=0, \infty$. Neglecting fast oscillations, $\left\langle\sigma_{r}^{z}(t) \sigma_{0}^{z}(t)\right\rangle_{c}$ increases as $r^{2}$ for $r<2 t$ and then it drops almost immediately to 0 . 
- The autocorrelation function (not connected) of $\sigma_{z}$ at different times is

$$
\left\langle\sigma_{0}^{z}\left(t_{1}\right) \sigma_{0}^{z}\left(t_{2}\right)\right\rangle=J_{0}^{2}\left(2 t_{2}-2 t_{1}\right)-\frac{1}{4}\left[f\left(t_{2}+t_{1}\right) \pm g\left(t_{2}-t_{1}\right)\right],
$$

where $f(x)=J_{2}(2 x)+J_{0}(2 x), g(x)=J_{2}(2 x)-J_{0}(2 x)$ and the sign $+(-)$ refers to $h_{0}=0\left(h_{0}=\infty\right)$.

Let us comment on these results in view of the general understanding we found so far. For $t<t^{*}$ all the connected correlation functions are zero, in agreement with CFT. All the oscillation terms of the asymptotic form $\cos (4 t)$ are, as we discussed in the previous section, a lattice effect. Concerning the $\sigma_{z}$ correlator, the $r^{2}$ dependence for $t>r / 2$ is a consequence of the fact that $\sigma_{z}$ is not primary. The same is true for the two-time correlations function of $\sigma_{z}$ that also decays as a power law of $t-s$ for large times, instead of the exponential prediction by CFT for primary field.

\section{HIGHER DIMENSIONS}

Until now we just considered one-dimensional systems. Despite the fact that in low dimensions the effect of fluctuations is more pronounced making the physics highly non-trivial it is desirable to have results in higher dimensions as well. The method presented in Sec. II to obtain the non-equilibrium dynamics of a quantum model close to a critical point from the critical behavior of a system confined in a slab geometry applies to generic dimension $d$ through the study of the hamiltonian (4). Its analysis proceeds via field-theoretical RG that may provide the all scaling quantities of the model in an expansion close to the upper critical dimensions (u.c.d.), that is $D=d+1=4$. Above the u.c.d. mean-field (or gaussian) results are exact, with logarithmic correction at the u.c.d.. For dimensions lower than the u.c.d., the scaling quantities are obtained as series in $\epsilon=4-(d+1)$. Thus for the time-evolution problem the simple mean-field solution represents an exact scaling result (a part log corrections) for the physically relevant three-dimensional case. An alternative method to attack analytically the hamiltonian (4) is to consider an $N$ component field $\phi$ and taking the limit $N \rightarrow \infty$, but this will not be employed here.

\section{A. Dirichlet boundary conditions: the two-point function}

The $D$-dimensional slab geometry with Dirichlet boundary conditions has been the subject of several investigations. The two-point function has been calculated at the first order in $\epsilon$ expansion in Ref. [43]. The gaussian two-point function, with partial Fourier transform in the parallel directions reads [43]

$$
G\left(p, z_{1}, z_{2}\right)=\frac{1}{2 b}\left(e^{-b\left|z_{1}-z_{2}\right|}-e^{-b\left(z_{1}+z_{2}\right)}+\frac{e^{-b\left(z_{1}-z_{2}\right)}+e^{-b\left(z_{2}-z_{1}\right)}-e^{-b\left(z_{1}+z_{2}\right)}-e^{b\left(z_{1}+z_{2}\right)}}{e^{2 b L}-1}\right),
$$

with $b=\sqrt{p^{2}+m^{2}}$. We are interested in the case where $L=2 \tau_{0}, z_{1}=z_{2}=\tau$ that we will analytically continue to $\tau \rightarrow \tau_{0}+i t$, and for computational simplicity we will restrict to the massless case $m=0$. Thus in real space and imaginary time, we have $(p=|\mathbf{p}|)$

$$
\begin{aligned}
G(r, \tau) & =\int \frac{d^{3} p}{(2 \pi)^{3}} e^{-i \mathbf{p} \cdot \mathbf{r}} \frac{1}{2 p}\left(1-e^{-2 p \tau}+\frac{2(1-\cosh (2 p \tau))}{e^{4 p \tau_{0}}-1}\right)= \\
& =\frac{1}{(2 \pi)^{2}} \int_{0}^{\infty} p^{2} d p \frac{1}{2 p}\left(1-e^{-2 p \tau}+\frac{2(1-\cosh (2 p \tau))}{e^{4 p \tau_{0}}-1}\right) \int_{-1}^{1} d(\cos \theta) e^{i p r \cos \theta}= \\
& =\frac{1}{(2 \pi)^{2} r} \int_{0}^{\infty} d p \sin p r\left(1-e^{-2 p \tau}+\frac{2(1-\cosh (2 p \tau))}{e^{4 p \tau_{0}}-1}\right) .
\end{aligned}
$$

This integral can be performed by making the sum over all the residues coming from the denominator $e^{4 p \tau_{0}}-1$. The calculation is rather involved, but the final result is very simple:

$$
G(r, \tau)=\frac{1}{\pi r \tau_{0}} \frac{\operatorname{coth}\left(\pi r / 4 \tau_{0}\right) \sin ^{2}\left(\pi \tau / 2 \tau_{0}\right)}{\cosh \left(\pi r / 2 \tau_{0}\right)-\cos \left(\pi \tau / \tau_{0}\right)} .
$$

Continuing to real time $\tau=\tau_{0}+i t$ we obtain

$$
G(r, t)=\frac{1}{\pi r \tau_{0}} \frac{\operatorname{coth}\left(\pi r / 4 \tau_{0}\right) \cosh ^{2}\left(\pi t / 2 \tau_{0}\right)}{\cosh \left(\pi r / 2 \tau_{0}\right)+\cosh \left(\pi t / \tau_{0}\right)},
$$


that for $t, r \gg \tau_{0}$ simplifies to

$$
G(r, t) \simeq \frac{1}{r \tau_{0}} \frac{e^{\pi t / \tau_{0}}}{e^{\pi t / \tau_{0}}+e^{\pi r / 2 \tau_{0}}} \propto \begin{cases}e^{\pi(t-r / 2) / 2 \tau_{0}} / r & \text { for } t<r / 2, \\ 1 / r & \text { for } t>r / 2 .\end{cases}
$$

We recall that this result in $d=3$ is exact a part log corrections. Thus the basic structure of the two-point function in $3 \mathrm{D}$ is the same as in $1 \mathrm{D}$, with a characteristic time $t^{*}=r / 2$. Using the result in Ref. [43] it is in principle possible to calculate the correlation functions for $d<3$ in the $\epsilon$ expansion framework. However, this requires the analytical continuation of complicated functions, resulting in a quite cumbersome algebra, as we shall see in the following for a simpler observable.

Eq. (74) can be used in principle to determine the gaussian behavior in any $d<3$. Unfortunately the integral one gets is not analytically tractable for $d \neq 1,3$. A possible strategy would be to perform the analytic continuation before of the integral and then evaluate it through a saddle-point approximation. It is straightforward to show that the result obtained in this way is equivalent to what we discuss in section VD where we remand for the analysis of $1<d<3$.

\section{B. Dirichlet boundary conditions: a non-trivial one-point function}

In the case of Dirichlet boundary conditions, the order parameter profile in the slab geometry is trivially vanishing. However not all the one-point expectation values are zero. Let us consider as a typical example the operator $\mathcal{O}=\phi^{2}$ that has been calculated at the first order in $\epsilon$ expansion in Ref. [44]. It has the scaling form

$$
\langle\mathcal{O}(z, L)\rangle \simeq L^{-d+1 / \nu} H(z / L),
$$

where $\nu$ is the correlation length exponent. In $D=4$, the function $H(x)$ is [44]

$$
H(x)=\frac{\pi^{2}}{\sin ^{2}(\pi x)}-\frac{\pi^{2}}{3},
$$

that, using $L=2 \tau_{0}$ and continuing to $z=\tau=\tau_{0}+i t$, leads to the real time evolution

$$
\langle\mathcal{O}(t)\rangle-\langle\mathcal{O}(t=\infty)\rangle \simeq \tau_{0}^{-2} \frac{\pi^{2}}{\cosh ^{2}\left(\pi t / 2 \tau_{0}\right)} \sim \tau_{0}^{-2} e^{-\pi t / 2 \tau_{0}},
$$

i.e. $\langle\mathcal{O}(t)\rangle$ approaches its asymptotic value exponentially, as it as been found for all primary operators in $d=1$.

The one-loop result for the $O(N)$ model in $D=4-\epsilon$ dimensions is [44]

$$
H(x)=\left[\frac{\pi^{2}}{\sin ^{2}(\pi x)}-\frac{\pi^{2}}{3}\right]\left(1+\epsilon \frac{N+2}{N+8} \ln \frac{\pi}{\sin \pi x}\right)-\epsilon\left[\zeta^{\prime}(2, x)+\zeta^{\prime}(2,1-x)\right]+\text { const },
$$

where const stands for terms do not depend on $x$ and $\zeta^{\prime}(\alpha, x)=\partial_{\alpha} \zeta(\alpha, x)$ and $\zeta$ is the generalized Riemann function. This example clearly shows that within the $\epsilon$ expansion, the scaling functions contain logarithmic contributions that originate from the expansion of power law as e.g. $\sin ^{\epsilon} \pi x=1+\epsilon \ln \sin \pi x$. To have a function with good analytical structure to perform the real time continuation, it is desirable to "exponentiate" such logarithms. In Ref. [44] the exponentiation procedure leaded to

$$
\begin{aligned}
H(x)=\left(\frac{\pi}{\sin (\pi x)}\right)^{2-1 / \nu}[\zeta(D-2, x)+\zeta(D-2,1-x)-2 \zeta(D-2)] & \\
& -\frac{\pi^{2}}{6}(2-1 / \nu)\left(\frac{\pi}{\sin (\pi x)}\right)^{D-2-1 / \nu},
\end{aligned}
$$

with $\nu=1 / 2+(N+2) /(N+8) \epsilon / 4$. Performing the analytical continuation we have the sum of two exponentials, and in any dimension $d<3$ the second one has a largest "relaxation time" that hence is dominating for large $t$. Considering only the second term we have

$$
\left\langle\mathcal{O}\left(t \gg \tau_{0}\right)\right\rangle-\langle\mathcal{O}(t=\infty)\rangle \propto e^{-(d-1-1 / \nu) \pi t / 2 \tau_{0}} .
$$

Note that the subleading term $e^{-(2-1 / \nu) \pi t / 2 \tau_{0}}$ is multiplied by a $\log t$ term arising from the $\zeta$ function.

This example put forward the idea that (at least for Dirichlet boundary conditions) the exponential relaxation of the one-point functions is not only a property of one-dimensional systems, but holds in any dimension (with eventually $\log$ corrections) with relaxation times related to the scaling dimensions of the operator. 


\section{Fixed boundary conditions: the one-point function}

The case of the extraordinary transition, that corresponds to fixed boundary conditions, has been considered in Ref. [45]. The magnetization profile can be written as [45]

$$
\phi(z)=\frac{2 K_{4}}{L} \frac{\operatorname{dn}\left(2 K_{4} z / L\right)}{\operatorname{sn}\left(2 K_{4} z / L\right)},
$$

where $K_{4}=K\left(k_{4}\right)$, where $K(k)$ is the elliptic integral, $k_{4}$ the elliptic modulus that in terms of the parameter of the model is $\phi^{2} L^{2}=\left(2 K_{4}\right)^{2}\left(2 k_{4}^{2}-1\right)$, and $\operatorname{sn}(x)$ and $\operatorname{dn}(x)$ and the Jacobi functions. Continuing to real time, and using the properties of the Jacobi functions we obtain

$$
\phi(t)=\sqrt{1-k_{4}} \operatorname{cn}\left(K_{4} t / \tau_{0}, 1-k_{4}\right),
$$

that, contrarily to all the other cases we have considered, is oscillating and not exponentially decreasing.

To our knowledge there are no result in the $\epsilon$-expansion for the magnetization profile. However, using "localfunctional methods" [46] it has been obtained an approximate profile in $D=3$ that involves, as in mean-field theory, Jacobi elliptic functions. It can be easily continued to real time via $z \rightarrow \tau_{0}+i t$ and again one finds an oscillating behavior with time. The method exploited in Ref. [46] can be used in any dimensions, obtaining an always an oscillating behavior with a period that diverges as $D$ approaches 2, recovering Eq. (7). All these calculations are essentially mean field and we do not know how the inclusion of fluctuations changes them. It can possible that for the extraordinary transition (i.e. fixed initial conditions), the exponential decay founds at $D=2$ is more an exception rather than a rule, because of the simple analytic structure of the trigonometric functions in the complex plane. Another possibility is that fluctuations destroy these oscillations. Only a complete analytical calculation (e.g. in large $N)$ can help in understanding this point.

\section{A real-time solvable model}

As for the one-dimensional case, it is worth to check the results coming from the analytical continuation of large imaginary-time with exactly solvable models. The simplest (and probably one of the few) model solvable in generic dimension is the generalization of the hamiltonian (38) to a $d$-dimensional hypercubic lattice. The solution of such model proceeds via Fourier transform as in one dimension. The final result is simply give by Eq. (53) with the replacement $d k \rightarrow d^{d} k$, i.e.

$$
\left\langle\varphi_{r}(t) \varphi_{0}(t)\right\rangle-\left\langle\varphi_{r}(0) \varphi_{0}(0)\right\rangle=\int_{\mathrm{BZ}} \frac{d^{d} k}{(2 \pi)^{d}} e^{i \mathbf{k} \cdot \mathbf{r}} \frac{\left(\Omega_{0 k}^{2}-\Omega_{k}^{2}\right)\left(1-\cos \left(2 \Omega_{k} t\right)\right)}{\Omega_{k}^{2} \Omega_{0 k}} .
$$

First of all let us note that this expression in virtually identical to Eq. (75) if we take $\tau_{0} \propto m_{0}^{-1} \rightarrow 0$. In fact, taking $\tau_{0} \rightarrow 0$ in Eq. (75), only the third term matters, since it is $O\left(\tau_{0}^{-1}\right)$ relative to the first two. Taking $z=r+i t$ and $b=\Omega_{p}$ we get $G(p) \propto \tau_{0}^{-1} \int \Omega_{p}^{-2}\left(1-\cos 2 \Omega_{p} t\right)$ that is Eq. (87) for $m_{0} \rightarrow \infty$.

To understand the general features let us consider in details the conformal evolution $\left(\Omega_{k}=v|\mathbf{k}|\right)$ from a disordered state $\left(\Omega_{0 k}=m_{0}\right)$. The derivative of the two-point function is $(k=|\mathbf{k}|)$

$$
\partial_{t}\left\langle\varphi_{r}(t) \varphi_{0}(t)\right\rangle=2 m_{0} \operatorname{Im} \int \frac{d^{d} k}{(2 \pi)^{d}} \frac{e^{i(\mathbf{k} \cdot \mathbf{r}-2 k v t)}}{k} .
$$

Except for $d=1$, this can be done analytically only in $d=3$, where we can write it as

$$
\sim \int k d k \int d \theta \sin \theta e^{i k(r \cos \theta-2 v t)} \sim \int d k(\sin (k r) / r) e^{2 i k v t} \sim(1 / r) \delta(v t-r / 2) .
$$

Integrating with respect to $t$ we get zero for $t<r / 2 v$ and $1 / r$ for $t>r / 2 v$.

For general $d$ we have

$$
\partial_{t}\left\langle\varphi_{r}(t) \varphi_{0}(t)\right\rangle \propto 2 m_{0} \operatorname{Im} \int k^{d-2} d k \int d \theta(\sin \theta)^{d-2} e^{i k(r \cos \theta-2 v t)} .
$$

By a saddle point argument, we can assume that the dominant behavior comes from $\theta$ close to zero, so the $\theta$ integral gives

$$
\int d \theta \theta^{d-2} e^{-i k r \theta^{2} / 2} \sim(k r)^{-(d-1) / 2}
$$


leading to

$$
\int d k \frac{k^{d-2}}{(k r)^{(d-1) / 2}} e^{i(k(r-2 v t))} \sim r^{-(d-1) / 2}(2 v t-r)^{-(d-1) / 2} \Theta(v t-r / 2) .
$$

Integrating with respect to $t$, we get zero for $t<r / 2 v$, as expected by causality, and

$$
r^{-(d-1) / 2}(2 v t-r)^{(3-d) / 2},
$$

for $t>r / 2 v$. It is interesting that this gaussian correlation function blows up at large $t$ only for $d<3$, when we expect the fluctuations to become important. It would be interesting to study this in the $\phi^{4}$ theory for large $N$, by replacing $\phi^{4}$ by $3\left\langle\phi^{2}\right\rangle \phi^{2}$ in the usual way, where now $\left\langle\phi^{2}\right\rangle$ depends on $t$ and is calculated self-consistently.

\section{PHYSICAL INTERPRETATION AND DISCUSSION}

In this paper we studied in general the non-equilibrium unitary dynamics that follows a sudden quantum quench. We showed that if the hamiltonian $H$ governing the time evolution is at a critical point, while $H_{0}$ (i.e. the one for $t<0$ ) is not, the expectation value of a class of operators (primary ones in CFT) relaxes to the ground-state value exponentially in time with universal ratio of decaying constants. We also found that connected two-point functions of operators at distance $r$ are vanishing for $t<r / 2 v$, while for $t>r / 2 v$ reach exponentially fast a value that depends exponentially on the separation, in contrast with the power laws typical of equilibrium configuration.

We also considered the real-time dynamics of simple exactly solvable models and we found that several of the typical characteristics of the critical points still hold. Roughly speaking, critical points are not special as far as quenching dynamics is concerned. In fact, also for gapped systems, connected correlation functions vanish (or are strongly suppressed) for $t<r / 2 v$ and for asymptotic large times resemble those at finite temperature despite the fact that the whole system is in a pure state. Several other examples in the recent literature (see e.g. $[9,15,24,26,39,41]$ ) gives further evidence that these two effects are actually true in general, at least in the realm of exactly solvable models considered so far.

In the following we give a simple interpretation of these two features separately trying to understanding their physical origin.

\section{A. The horizon effect}

The qualitative, and many of the quantitative, features found for the time evolution of correlation functions may be understood physically on the basis of a picture we first introduced in Ref. [39] to describe the time evolution of the entanglement entropy. Later we generalized it to correlation functions in Ref. [33] and it has been largely adopted thereafter $[2,24,26,41]$.

We emphasize that such scheme is not an ab initio calculation but rather a simplified picture which allows us to explain physically our findings. The initial state $\left|\psi_{0}\right\rangle$ has an (extensively) high energy relative to the ground state of the hamiltonian $H$ which governs the subsequent time evolution, and therefore acts as a source of quasiparticle excitations. Those quasi-particles originating from closely separated points (roughly within the correlation length $\xi_{0}=m_{0}^{-1}$ of the ground state of $H_{0}$ ) are quantum entangled and particles emitted from far different points are incoherent. If the quasiparticle dispersion relation is $E=\Omega_{k}$, the classical velocity is $\mathbf{v}_{k}=\nabla_{k} \Omega_{k}$. We assume that there is a maximum allowed speed $v_{m}=\max _{\mathbf{k}}\left|\mathbf{v}_{\mathbf{k}}\right|$. A quasiparticle of momentum $k$ produced at $\mathbf{r}$ is therefore at $\mathbf{r}+\mathbf{v}_{k} t$ at time $t$, ignoring scattering effects. This is the only physical assumption of the argument. Scattering effects are not present in the theories considered so far, but as evident from the argument outlined below they can play a role for only $t>r / 2 v_{m}$ (allowing, perhaps, for a renormalization of $v_{m}$ by the interactions).

These free quasi-particles have two distinct effects. Firstly, incoherent quasi-particles arriving a given point $\mathbf{r}$ from well-separated sources cause relaxation of (most) local observables at $\mathbf{r}$ towards their ground state expectation values. (An exception is the local energy density which of course is conserved.) Secondly, entangled quasi-particles arriving at the same time $t$ at points with separation $|\mathbf{r}| \gg \xi_{0}$ induce quantum correlations between local observables. In the case where they travel at a unique speed $v$ (as in CFT), therefore, there is a sharp "horizon" or light-cone effect: the connected correlations do not change from their initial values until time $t \sim|\mathbf{r}| / 2 v$. In the CFT case this horizon effect is rounded off in a (calculable) manner over the region $t-|\mathbf{r}| / 2 v \sim \tau_{0}$, since quasi-particles remain entangled over this distance scale. After this they rapidly saturate to time-independent values. For large separations (but still $\ll 2 v t$ ), these decay exponentially $\sim \exp \left(-\pi x|\mathbf{r}| / 2 \tau_{0}\right)$. Thus, while the generic one-point functions relax to their ground-state values (we recall in CFT this relaxation is exponential $\sim \exp \left(-\pi x v t / \tau_{0}\right)$ ), the correlation functions do not, because, 
at quantum criticality, these would have a power law dependence. Of course, this is to be expected since the mean energy is much higher than that of the ground state, and it does not relax.

This simple argument also explains why for the case of a semi-infinite chain the relevant time scale is $r / v$ rather than $r / 2 v$, since one of the two particles arriving in $r$ has been reflected from the end of the chain. This has been also stressed in Ref. [41], in the study of the time dependence of entanglement entropy of finite chains with open boundary conditions.

All our results are consistent with this picture as long as the quasi-particles are assumed to all propagate at the same speed, resulting from a "conformal" dispersion relation $\Omega_{k}=v|k|$. However, it is very simple to generalize this picture to different dispersion relations, taking into account that each particle propagates at group velocity $v_{k} \equiv \Omega_{k}^{\prime}$ appropriate to the wave number $k$. In this case the horizon effect first occurs at time $t \sim|\mathbf{r}| / 2 v_{m}$, where $v_{m}$ is the maximum group velocity. If $v_{m}$ occurs at a non-zero wave number, it gives rise to spatial oscillations in the correlation function. Thus again connected correlation function are expected to be strongly suppressed for $t<t^{*}$ and start developing only after $t^{*}$. In the case of a general dispersion relation we do not have a proof, beyond the stationary phase approximation, that the connected correlation functions remain constant up to this time, but one would expect it on the grounds of causality. (The proof in the case of a relativistic dispersion relation uses Lorentz invariance in an essential way.) However, because there are also quasi-particles moving at speeds less than $v_{m}$, the approach to the asymptotic behavior at late times is less abrupt. In fact, for a lattice dispersion relation where $\Omega_{k}^{\prime}$ vanishes at the zone boundary, the approach to the limit is slow, as an inverse power of $t$. A similar result applies to the 1-point functions. This is consistent with the exact results obtained here and elsewhere.

\section{B. The large time limit and the generalized Gibbs ensemble}

The existence and the understanding of the asymptotic state resulting from the evolution from an arbitrary state is one of the most-interesting problem in statistical mechanics. A robust theory able to predict this state ab-initio still does not exist. A currently popular idea is that for late times the system (or rather macroscopically large subsystems) 'look like' they are in a thermal state, despite the fact that the actual state of the whole system is pure. A common belief is that a region of dimension $r$ can be thermalized by the infinitely large rest of the system which acts as a bath (see e.g. [24, 39, 47]). But this intriguing idea is not sufficient to give the value of the resulting effective temperature.

A major step toward the clarification of the properties of the asymptotic state has been made by Rigol et al. [15]. In fact, it was conjectured that if the asymptotic stationary state exists, it is given by a generalized Gibbs ensemble obtained by maximizing the entropy $S=-\operatorname{Tr} \rho \log \rho$, subject to all the constraints imposed by the dynamics [15]. Consequently, denoting with $I_{m}$ a maximal set of commuting and linearly independent integrals of motion, the density matrix is

$$
\rho=Z^{-1} e^{-\sum_{m} \lambda_{m} I_{m}}, \quad Z=\operatorname{Tr} e^{-\sum_{m} \lambda_{m} I_{m}} .
$$

We note that such a density matrix describes a pure state only if the model under consideration is integrable, i.e. if the number of integral of motions equals the number degrees of freedom. If there are not enough integrals of motion, $\rho$ corresponds to a mixed state and it is not clear to us to which extent it can describe the pure state resulting from the time-evolution. The values of the Lagrange multipliers $\lambda_{m}$ are fixed by the initial conditions:

$$
\operatorname{Tr} I_{m} \rho=\left\langle I_{m}\right\rangle_{t=0} .
$$

In the following we will take this generalized Gibbs ensemble as a postulate and we will show how it nicely and naturally explains the "effective temperature" effect observed for large times. However we stress that there is still no proof for this assumption that, to our opinion, cannot be considered on the same fundamental level as the thermal Gibbs ensemble.

Let us consider the chain of harmonic oscillators of the previous section as a typical example. We will soon see that most of the features are quite general. In this case the natural choice for an infinite set of integral of motion is the number of particles with momentum $k$, i.e. $n_{k}=A_{k}^{\dagger} A_{k}$. Most other observables can be written in terms of these, i.e. $H=\sum_{k} \Omega_{k} n_{k}$. Consequently the expectation value is given by

$$
\langle\mathcal{O}\rangle_{t=\infty}=\operatorname{Tr} \mathcal{O} \rho=\operatorname{Tr} \mathcal{O} Z^{-1} e^{-\sum_{k} \lambda_{k} n_{k}},
$$

that can be seen as a thermal density matrix with a $k$ dependent effective temperature given by

$$
\beta_{\text {eff }}(k) \Omega_{k}=\lambda_{k} .
$$

Thus an effective temperature already appeared. Note that this state can still be pure because such a temperature is $k$ dependent. 
To fix $\lambda_{k}$ we need $\left\langle n_{k}\right\rangle_{t=0}$. From Eq. (50) we get

$$
\left\langle n_{k}\right\rangle_{t=0}=\left\langle A_{k}^{\dagger} A_{k}\right\rangle_{t=0}=d_{k}^{2}=\frac{1}{4}\left(\frac{\Omega_{k}}{\Omega_{0 k}}+\frac{\Omega_{0 k}}{\Omega_{k}}\right)-\frac{1}{2} .
$$

The calculation then proceeds as for a thermal distribution

$$
\left\langle n_{k}\right\rangle_{\rho}=\operatorname{Tr} n_{k} \rho=-\frac{\partial}{\partial \lambda_{k}} \ln Z, \quad \text { with } \quad Z=\operatorname{Tr} e^{-\sum_{k} \lambda_{k} n_{k}}=\prod_{k} \sum_{n_{k}=0}^{\infty} e^{-\lambda_{k} n_{k}}=\prod_{k} \frac{1}{1-e^{-\lambda_{k}}},
$$

so that

$$
\left\langle n_{k}\right\rangle_{\rho}=\frac{\partial}{\partial \lambda_{k}} \sum_{k} \ln \left(1-e^{-\lambda_{k}}\right)=\frac{1}{e^{\lambda_{k}}-1}
$$

From this $e^{\lambda_{k}}=1+d_{k}^{-2}$ and

$$
\beta_{\text {eff }}(k)=\frac{\ln \left(1+d_{k}^{-2}\right)}{\Omega_{k}} .
$$

At finite temperature the correlation function in momentum space is

$$
\left\langle\varphi_{k} \varphi_{-k}\right\rangle_{\beta}=\left\langle\frac{2}{\Omega_{k}}\left(A_{k}+A_{k}^{\dagger}\right)\left(A_{-k}+A_{-k}^{\dagger}\right)\right\rangle_{\beta}=\frac{2}{\Omega_{k}}\left(\left\langle A_{k}^{\dagger} A_{-k}\right\rangle_{\beta}+\left\langle A_{k} A_{-k}^{\dagger}\right\rangle_{\beta}\right)=\frac{2}{\Omega_{k}} \frac{1+e^{-\beta \Omega_{k}}}{1-e^{-\beta \Omega_{k}}},
$$

that substituting the previous result for $\beta_{\text {eff }}$ gives

$$
\rho(k)_{t=\infty}=\left\langle\varphi_{k} \varphi_{-k}\right\rangle_{t=\infty}=\frac{2}{\Omega_{k}}\left(1+2 d_{k}^{2}\right)=\frac{\Omega_{k}^{2}+\Omega_{0 k}^{2}}{\Omega_{k}^{2} \Omega_{0 k}},
$$

that is exactly the time-average of Eq. (54) reproducing, after Fourier transforming, the well defined correlation in real space for $t \rightarrow \infty$.

So this generalized Gibbs ensemble correctly reproduces the exact diagonalization of the model and gives also few insights more. In fact, in the limit $m_{0} \rightarrow \infty$ the effective temperature is independent from $k$ and $m$ obtaining $\beta_{\text {eff }}=4 / m_{0}$, explaining a posteriori the simplicity of the results in this case. Note instead that for arbitrary $m_{0}$ and $m=0$, i.e. conformal evolution, $\beta_{\mathrm{eff}}(k)$ is a function of $k$. In this case, the large distance properties of correlation functions are described by the mode with $k=0$, for which, independently of $m_{0}$ we get $\beta_{\text {eff }}(k=0, m=0)=4 / m_{0}$, consistently with the previous findings and general expectations. Furthermore we can conclude that the large $r$ asymptotic behavior is always governed by the effective temperature $\beta_{\text {eff }}(k=0)=2\left(\log \left(\left|m_{0}-m\right| /\left(m_{0}+m\right)\right) / m\right.$. Another interesting feature is that $\beta_{\mathrm{eff}}(k=0)$ gives the asymptotic behavior of the correlation functions of all those observables that are effectively coupled with the zero-mode. In the opposite case the relevant temperature is the largest $\beta_{\text {eff }}(k)$ with the $k$ mode coupled with the observable. Another interesting feature is that on a very rough basis one can be tempted to assume that that $\beta_{\text {eff }}(k)$ is directly related to the excess of energy of the mode $k$ generated by the quench, but this is not the case. In fact $\langle H\rangle_{t=0}=\sum_{k} \Omega_{k} d_{k}^{2}$ that is different from $\beta_{\text {eff }}^{-1}$, being the same only in the limit $d_{k}^{-2} \rightarrow 0$, i.e. $m_{0} \rightarrow \infty$.

Clearly the same reasoning of before applies every time we consider a model that can be diagonalized in momentum space with a proper choice of the quasi-particles, i.e. every time that $H=\sum_{k} \Omega_{k} A_{k}^{\dagger} A_{k}$ for some $A_{k}$. This means that the excitations are non-interacting. As far as we are aware all the applications of the generalized Gibbs ensemble to the date only concern this kind of models $[15,26]$, but there are few numerical hints suggesting that can be true more generically [29].

Now we outline how we imagine the generalized Gibbs ensemble given by Eq. (94) could be used to justify the effective temperature scenario for large time for any integrable system, i.e. with a complete set of integrals of motion. The hamiltonian can be written as $H=\sum_{m} a_{m} I_{m}$, with some $a_{m}$ eventually zero. Thus one can think to an $m$ dependent effective temperature $\beta_{\text {eff }}(m)=\lambda_{m} / a_{m}$, but this temperature does not give directly the behavior of the correlation function for large distance because in general the integral of motions are not diagonal in $k$-space. Thus we can only conjecture that the correlation functions of a given operator $O(r)$ are governed by the largest $\beta_{\text {eff }}(m)$ with $m$ among the integral of motions to which $O(r)$ effectively couples. We stress that this is only a crude argument and we are still not able to put it on a firmer basis. 


\section{Discussion and open questions}

We presented a quite complete picture on the time evolution of a quantum system after a sudden quench of one hamiltonian parameter. Despite the fact that a lot of work has been done, still more is left for future investigation.

The first problem that must be addressed is the real-time dynamics of effectively interacting systems. In this direction Bethe Ansatz solvable models like the Lieb-Liniger gas and Heisenberg spin chains are among the best candidates for an analytical approach. This would clarify how a non-trivial two-body scattering matrix can modify the time-evolution (only inside the light-cone) of the correlation functions and whether the crude argument we outlined for an "effective temperature" for the long-time state is valid. To clarify this point also the study of (boundary) integrable massive field theories can be of some help.

Another approach, currently under investigation[48], is the direct analysis of the perturbative expansion for the correlation functions in a $\lambda \phi^{4}$ field theory. This has some simplifying features in the large $m_{0}$ limit which may allow it to be resummed to all orders. It is very important to get non-trivial results for such a non-integrable interacting theory.

Numerical computations for non-integrable systems should also be performed to understand if and eventually to which extent the generalized Gibbs ensemble picture is valid beyond integrability. Some numerics concerning this point are already available $[22,23,29]$ but still a clear scenario is not emerged. Among non-integrable models special care must be given to disordered systems because of the non existence of a speed of sound as a consequence of Anderson localization [49]. Some insights can be obtained from those models whose equilibrium behavior can be analytically obtained by means of the strong-disorder renormalization-group [50]. Even in this case time-dependent DMRG can help a general understanding. The time-evolution of the entanglement entropy [41] already revealed that in these systems there is no sign of the light-cone (as easily predictable because of the absence of speed of sound) and the effective motion of "quasi-particles" is more diffusive rather than ballistic. Furthermore the results at the largest available times does not look thermal at all, but this can be also due to the time window accessible with numerics.

Another very interesting question is what happens at finite temperature and under what conditions a system can equilibrate. It should be relatively simple to generalize the results for all the "quasi-free" models already considered at $T=0$. Work in this direction is in progress, but it will be almost impossible to consider the same problem for more complicated models.

Finally it is also important to understand the role played by the initial state. We assumed always a translational invariant one with short range correlations. Thus one natural modification consists in taking a state that is only locally different from the actual ground-state. This problem is known in the literature as a "local quench" and it has already been considered to some extent (see e.g. [51-53]), but still a general picture as the one outlined here for global quenches does not exist (this is also interesting for eventual connections with quantum impurity problems, see [54] as a review). Another natural modification of the initial state is one with long-range correlations, such as a critical one and let it evolve with another critical hamiltonian. This has been done for the Luttinger liquid [26] and the results show the typical functional dependence of a light-cone scenario (i.e. everything depends only on $x \pm 2 v t$ ) but the long-time correlations decays as power-laws, with exponents that are different from equilibrium ones and can be predicted by the generalized Gibbs ensemble.

\section{Acknowledgments}

This work was supported in part by EPSRC grants GR/R83712/01 and EP/D050952/1. This work has been finished when PC was a guest of the Institute for Theoretical Physics of the Universiteit van Amsterdam. This stay was supported by the ESF Exchange Grant 1311 of the INSTANS activity.

[1] M. Greiner, O. Mandel, T. W. Hänsch, and I. Bloch, Collapse and Revival of the Matter Wave Field of a Bose-Einstein Condensate, 2002 Nature (London) 41951 [cond-mat/0207196]

[2] L. E. Sadler, J. M. Higbie, S. R. Leslie, M. Vengalattore, and D. M. Stamper-Kurn, Spontaneous symmetry breaking in a quenched ferromagnetic spinor Bose-Einstein condensate, 2006 Nature 443, 312 A. Lamacraft, Quantum quenches in a spinor condensate, 2006 Phys. Rev. Lett. 98, [cond-mat/0611017].

[3] B. Paredes, A. Widera, V. Murg, O. Mandel, S. Falling, I. Cirac, G. V. Shlyapnikov, T. W. Hansch, and I. Bloch, TonksGirardeau gas of ultracold atoms in an optical lattice, 2004 Nature 429, 277;

T. Kinoshita, T. Wenger, and D. S. Weiss, Observation of a One-Dimensional Tonks-Girardeau Gas, 2004 Science 305, 1125 .

[4] T. Kinoshita, T. Wenger, and D. S. Weiss, A quantum Newton's cradle, 2006 Nature 440, 900. 
[5] C. Orzel, A. K. Tuchman, M. L. Fenselau, M. Yasuda, and M. A. Kasevich, Squeezed States in a Bose-Einstein Condensate, 2001 Science 2912386

A. Widera, F. Gerbier, S. Fölling, T. Gericke, O. Mandel, and I. Bloch, Coherent Collisional Spin Dynamics in Optical Lattices, 2005 Phys. Rev. Lett. 95, 190405.

[6] G. Vidal, Efficient simulation of one-dimensional quantum many-body systems, 2004 Phys. Rev. Lett. 93, 040502 [quant$\mathrm{ph} / 0310089]$

A. J. Daley, C. Kollath, U. Schollwoeck, and G. Vidal, Time-dependent density-matrix renormalization-group using adaptive effective Hilbert spaces, 2004 J. Stat. Mech. P04005 [cond-mat/0403313];

S. R. White and A. E. Feiguin, Real time evolution using the density matrix renormalization group, 2004 Phys. Rev. Lett. 93, 076401 [cond-mat/0403310];

F. Verstraete, J. J. Garcia-Ripoll, and J. I. Cirac, Matrix Product Density Operators: Simulation of finite-T and dissipative systems, 2004 Phys. Rev. Lett. 93, 207204 [cond-mat/0406426];

D. Gobert, C. Kollath, U. Schollwoeck, and G. Schuetz Real-time dynamics in spin-1/2 chains with adaptive time-dependent DMRG, 2005 Phys. Rev. E 71, 036102 [cond-mat/0409692]

[7] E. Barouch and B. McCoy, Statistical Mechanics of the XY Model. I. 1970 Phys. Rev. A 21075

[8] E. Barouch and B. McCoy, Statistical Mechanics of the XY Model. II. Spin-Correlation Functions, 1971 Phys. Rev. A 3 786

E. Barouch and B. McCoy, Statistical Mechanics of the XY Model. III, 1971 Phys. Rev. A 32137

[9] F. Igloi and H. Rieger, Long-Range Correlations in the Nonequilibrium Quantum Relaxation of a Spin Chain, 2000 Phys. Rev. Lett. 853233 [cond-mat/0003193]

[10] L. Amico and A. Osterloh, Out of equilibrium correlation functions of quantum anisotropic XY models: one-particle excitations, 2004 J. Phys. A 37291 [cond-mat/0306285]

[11] K. Sengupta, S. Powell, and S. Sachdev, Quench dynamics across quantum critical points, 2004 Phys. Rev. A 69053616 [cond-mat/0311355]

[12] R. W. Cherng and L. S. Levitov, Entropy and Correlation Functions of a Driven Quantum Spin Chain, 2006 Phys. Rev. A 73, 043614 [cond-mat/0512689].

[13] A. Das, K. Sengupta, D. Sen, and B. K. Chakrabarti, Infinite-range Ising ferromagnet in a time-dependent transverse field: quench and ac dynamics near the quantum critical point, 2006 Phys. Rev. B 74, 144423 [cond-mat/0606137].

[14] T. S. Cubitt and J.I. Cirac, Engineering correlation and entanglement dynamics in spin systems, quant-ph/0701053.

[15] M. Rigol, V. Dunjko, V. Yurovsky, and M. Olshanii, Relaxation in a Completely Integrable Many-Body Quantum System: An Ab Initio Study of the Dynamics of the Highly Excited States of Lattice Hard-Core Bosons, 2007 Phys. Rev. Lett. 98, 50405 [cond-mat/0604476].

[16] A. del Campo and J. G. Muga, Dynamics of a Tonks-Girardeau gas released from a hard-wall trap, 2006 Europhys. Lett. 74, 965 [cond-mat/0511747];

A. Ruschhaupt, A. del Campo, and J. G. Muga, Momentum interferences of a freely expanding Bose-Einstein condensate in 1D due to interatomic interaction change, 2006 Europhys. J. D 40, 399 [cond-mat/0601437]

[17] K. Rodriguez, S.R. Manmana, M. Rigol, R.M. Noack, and A. Muramatsu, Coherent matter waves emerging from Mottinsulators, 2006 New J. Phys. 8, 169 [cond-mat/0606155].

[18] M. Rigol, A. Muramatsu, and M. Olshanii, Hard-core bosons on optical superlattices: Dynamics and relaxation in the superfluid and insulating regimes, 2006 Phys. Rev. A 74, 053616 [cond-mat/0612415].

[19] A. K. Tuchman, C. Orzel, A. Polkovnikov, M. A. Kasevich, Non-equilibrium coherence dynamics of a soft boson lattice, cond-mat/0504762.

[20] G.P. Berman, F.Borgonovi, F.M. Izrailev, A.Smerzi, Irregular Dynamics in a One-Dimensional Bose System, 2004 Phys. Rev. Lett. 92, 030404 [cond-mat/0309459].

[21] A. Minguzzi and D.M. Gangardt Exact coherent states of a harmonically confined Tonks-Girardeau gas, 2005 Phys. Rev. Lett. 94, 240404 [cond-mat/0504024].

[22] C. Kollath, U. Schollwck, J. von Delft, and W. Zwerger, One-dimensional density waves of ultracold bosons in an optical lattice, 2005 Phys. Rev. A 71, 053606 [cond-mat/0411403]

[23] C. Kollath, A. Laeuchli, and E. Altman; Quench dynamics and non equilibrium phase diagram of the Bose-Hubbard model, cond-mat/0607235.

[24] M. Cramer, C.M. Dawson, J. Eisert, and T.J. Osborne, Quenching, relaxation, and a central limit theorem for quantum lattice systems, cond-mat/0703314.

[25] E. Altman and A. Auerbach, Oscillating Superfluidity of Bosons in Optical Lattices, 2002 Phys. Rev. Lett. 89, 250404 [cond-mat/0206157].

[26] M. A. Cazalilla. Effect of suddenly turning on the interactions in the Luttinger model, 2006 Phys. Rev. Lett. 97, 156403 [cond-mat/0606236].

[27] E. Perfetto, Time-dependent evolution of two coupled Luttinger liquids, 2006 Phys. Rev. B 74, 205123 [cond-mat/0607720].

[28] V. Gritsev, E. Demler, M. Lukin, and A. Polkovnikov, Analysis of quench dynamics of coupled one dimensional condensates using quantum sine Gordon model, cond-mat/0702343.

[29] S.R. Manmana, S. Wessel, R.M. Noack, and A. Muramatsu Strongly correlated fermions after a quantum quench, condmat/0612030.

[30] E. A. Yuzbashyan, B. L. Altshuler, V. B. Kuznetsov, V. Z. Enolskii, Nonequilibrium Cooper pairing in the nonadiabatic regime, 2005 Phys. Rev. B 72, 220503(R) [cond-mat/0505493];

E. A. Yuzbashyan, O. Tsyplyatyev, and B. L. Altshuler, Relaxation and persistent oscillations of the order parameter in 
fermionic condensate, 2006 Phys. Rev. Lett. 96, 097005;

R. A. Barankov and L. S. Levitov, Synchronization in the BCS Pairing Dynamics as a Critical Phenomenon, 2006 Phys. Rev. Lett. 96, 230403 [cond-mat/0603317];

E. A. Yuzbashyan and M. Dzero, Dynamical vanishing of the order parameter in a fermionic condensate, 2006 Phys. Rev. Lett. 96, 230404 [cond-mat/0603404].

[31] E. Altman and A. Vishwanath, Dynamic projection on Feshbach molecules: a probe of pairing and phase fluctuations, 2005 Phys. Rev. Lett. 95, 110404 [cond-mat/0501683];

R. A. Barankov and L. S. Levitov, Dynamical projection of atoms to Feshbach molecules at strong coupling, condmat/0506323

[32] W. H. Zurek, U. Dorner, and P. Zoller, Dynamics of a Quantum Phase Transition, 2005 Phys. Rev. Lett. 95 105701 [cond-mat/0503511];

A. Polkovnikov, Universal adiabatic dynamics across a quantum critical point, 2005 Phys. Rev. B 72, 161201(R) [condmat/0312144];

J. Dziarmaga, Dynamics of a quantum phase transition in the random Ising model, 2006 Phys. Rev. B 74, 64416 [condmat/0603814];

L. Cincio, J. Dziarmaga, M. M. Rams, and W. H. Zurek, Entropy of entanglement and correlations induced by a quench: Dynamics of a quantum phase transition in the quantum Ising model, cond-mat/0701768.

[33] P. Calabrese and J. Cardy, 2006 Phys. Rev. Lett. 96, 136801 [cond-mat/0601225].

[34] H. W. Diehl, The theory of boundary critical phenomena, 1986 in Phase Transitions and Critical Phenomena vol 10 ed C Domb and J L Lebowitz (London: Academic)

H. W. Diehl, The theory of boundary critical phenomena, 1997 Int. J. Mod. phys. B 11, 3503 [cond-mat/9610143]

[35] J. L. Cardy, Conformal Invariance and Surface Critical Behavior, 1984 Nucl. Phys. B 240514

[36] J. L. Cardy, Boundary Conformal Field Theory, in Encyclopedia of Mathematical Physics, ed J.-P. Francoise, G. Naber, and S. Tsun Tsou, (Elsevier, Amsterdam, 2006).

[37] J. Cardy and D. Lewellen, Bulk and Boundary Operators in Conformal Field Theory, 1991 Phys. Lett. B 259 274

[38] H. Blöte, J. Cardy, and P. Nightingale, Conformal Invariance, the Central Charge, and Universal Finite Size Amplitudes at Criticality, 1986 Phys. Rev. Lett. 56, 742.

[39] P. Calabrese and J. Cardy, Evolution of Entanglement entropy in one dimensional systems, 2005 J. Stat. Mech. P04010 [cond-mat/0503393].

[40] P. Calabrese and J. Cardy, Entanglement entropy and quantum field theory, 2004 J. Stat. Mech. P06002 [hep-th/0405152]

[41] G. De Chiara, S. Montangero, P. Calabrese, and R. Fazio Entanglement Entropy dynamics in Heisenberg chains, 2006 J. Stat. Mech. P03001 [cond-mat/0512586]

[42] http://functions.wolfram.com/HypergeometricFunctions/MeijerG1/

[43] R. Klimpel and S. Dietrich, Structure Factor of thin films near continuous phase transitions, 1999 Phys. Rev. B 6016977

[44] M. Krech, E. Eisenriegler, and S. Dietrich, Energy density profiles in critical films, 1995 Phys. Rev. E 521345

[45] M. Krech, Casimir force in binary liquid mixtures, 1997 Phys. Rev. E 561642 and references therein.

[46] Z. Borjan and P. J. Upton, Order parameter profiles and Casimir amplitudes in critical slabs, 1998 Phys. Rev. Lett. 81 4911

[47] J. Eisert and T. J. Osborne, General Entanglement Scaling Laws from Time Evolution, 2006 Phys. Rev. Lett. 97, 150404 [quant-ph/0603114];

S. Bravyi, M. B. Hastings, and F. Verstraete, Lieb-Robinson Bounds and the Generation of Correlations and Topological Quantum Order, 2006 Phys. Rev. Lett. 97, 050401 [quant-ph/0603121].

[48] S. Sotiriadis and J. Cardy, in progress.

[49] C. K. Burrell and T. J. Osborne, Bounds on Information Propagation in Disordered Quantum Spin Chains, quantph/0703209.

P. W. Anderson, Absence of Diffusion in Certain Random Lattices, 1959 Phys. Rev. 109, 1492

[50] F. Igloi and C. Monthus, Strong disorder RG approach of random systems, 2005 Physics Reports 412,277 [condmat/0502448].

[51] L. Amico, A. Osterloh, F. Plastina, R. Fazio, and G. M. Palma, Dynamics of Entanglement in One-Dimensional Spin Systems, 2004 Phys. Rev. A. 69, 022304 [quant-ph/0307048]

[52] C. Kollath, U. Schollwoeck, and W. Zwerger, Spin-charge separation in cold Fermi-gases: a real time analysis, 2005 Phys. Rev. Lett. 95, 176401 [cond-mat/0504299]

[53] V. Eisler and I. Peschel, Evolution of entanglement after a local quench, cond-mat/0703379.

[54] H. Saleur, Lectures on Non Perturbative Field Theory and Quantum Impurity Problems, cond-mat/9812110. 